\title{
P-flag spaces and incidence stratifications
}

\author{
Davide Bolognini] Paolo Sentinell:2,
}

\begin{abstract}
For any finite poset $P$, we introduce a homogeneous space as a quotient of the general linear group. When $P$ is a chain this quotient is a complete flag variety. Moreover, we provide partitions for any set in a projective space, induced by the action of incidence groups of posets. Our general framework allows to deal with several combinatorial and geometric objects, unifying and extending different structures such as Bruhat orders, parking functions and weak orders on matroids. We introduce the notion of $P$-flag matroid, extending flag matroids.
\end{abstract}

\section{Introduction}

Flag varieties are classical homogeneous spaces, studied from several different points of view. They parameterize the flags of $V$, i.e. sequences of subspaces $V_{1} \subseteq \ldots \subseteq V_{n}=V$, where $V$ is an $n$-dimensional $\mathbb{F}$-vector space and $V_{i} \subseteq V$ is an $i$-dimensional subspace of $V$. They can be obtained as quotients of the general linear group $G L(n, \mathbb{F})$ with the subgroup $B$ of invertible upper triangular matrices. The action of $B$ on the flags gives rise to a partition into Schubert cells. Their Zariski closures are the so-called Schubert varieties, which are in bijection with the symmetric group $S_{n}$. The Bruhat order on $S_{n}$ is the poset of Schubert varieties ordered by inclusion. Similar facts hold for the Grassmannian $\operatorname{Gr}_{\mathbb{F}}(k, n)$, replacing $S_{n}$ with the subset $S_{n}^{(k)}$ of Grassmannian permutations.

In this article we introduce a new class of homogeneous spaces, namely the quotients $\mathrm{Fl}_{\mathrm{P}}(\mathbb{F}):=\mathrm{GL}(n, \mathbb{F}) / I^{*}(P ; \mathbb{F})$, where $I^{*}(P ; \mathbb{F})$ is the so-called incidence group of a poset $P=\left(\{1, \ldots, n\}, \leqslant_{P}\right)$, i.e. the group of invertible elements of the incidence algebra of $P$. Since the Borel subgroup $B$ is the group $I^{*}\left(c_{n} ; \mathbb{F}\right)$, where $c_{n}$ is the chain on $n$ elements, we recover classical flag varieties.

In Definition 3.1 we introduce $P$-flags in $V$. They are tuples $\left(V_{1}, \cdots, V_{n}\right)$ of vector subspaces of $V$, satisfying, among others, the following properties (see Proposition 3.3):

- $V_{i} \subseteq V_{j}$ if and only if $i \leqslant_{P} j$;

- $\operatorname{dim}\left(V_{i}\right)=\left|i^{\downarrow}\right|$, where $i^{\downarrow}=\left\{j \in[n]: j \leqslant{ }_{P} i\right\}$.

We prove that $\mathrm{Fl}_{\mathrm{P}}(\mathbb{F})$ is a homogeneous space parametrizing $P$-flags in $V$ (Theorem 3.9). For this reason, we call $\mathrm{Fl}_{\mathrm{P}}(\mathbb{F})$ the $P$-flag space over $\mathbb{F}$. The elements of these spaces are certain spanning subspace configurations, see Remark 3.15 .

\footnotetext{
${ }^{1}$ Dipartimento di Matematica, Università di Bologna, Bologna, Italy, davide.bolognini.cast@gmail.com

${ }^{2}$ Dipartimento di Matematica, Politecnico di Milano, Milan, Italy, paolosentinelli@gmail.com
} 
The second main contribution of this paper is a new tool to obtain finite partitions of any subset $X$ of a projective space, introducing the notion of incidence stratifications, see Definition 4.6. In fact, an incidence group $I^{*}(Q ; \mathbb{F})$, where $Q$ is a poset of cardinality $n$, acts on the projective space $\mathbb{P}\left(\mathbb{F}^{n}\right)$ by left multiplication; the orbits of this action are indexed by non-empty order ideals of $Q$ (Theorem 4.2).

This general framework allows to deal with several combinatorial and geometric objects, unifying and extending different structures such as Bruhat orders, parking functions and weak orders on matroids.

For the Grassmannian $\operatorname{Gr}_{\mathbb{F}}(k, n) \hookrightarrow \mathbb{P}\left(\wedge^{k} V\right)$, we consider a suitable poset $Q_{<}^{k}$ (Definition 4.8) to realize an incidence stratification. In this setting, for $Q=c_{n}$, we recover the classical Schubert cells (Proposition 4.16). When $Q=t_{n}$, the incidence group $I^{*}\left(\left(t_{n}\right)_{<}^{k} ; \mathbb{F}\right)$ is a maximal torus and we obtain the matroid strata introduced in [14] and studied, e.g. in [11, [12, [29], 31. See also [2, Section 2.4] and references there.

We introduce a poset $Q^{P}$ (Definition 5.1) to provide an incidence stratification of the $P$-flag space $\mathrm{Fl}_{\mathrm{P}}(\mathbb{F}) \hookrightarrow \mathbb{P}\left(\bigotimes_{i=1}^{n} \wedge^{\left|i_{P}^{\downarrow}\right|} V\right)$. In this way the Schubert stratification of a flag variety is recovered, see Proposition 5.5 .

One more contribution is the construction, for $\mathrm{Fl}_{\mathrm{P}}(\mathbb{F})$, of the $Q$-Bruhat poset, whose elements are order ideals of $Q^{P}$ (Definition 5.6). In a completely combinatorial way, we obtain the Bruhat order of $S_{n}$ as the $c_{n}$-Bruhat order on the classical flag variety, see Proposition 5.16. The study of the $t_{n}$-Bruhat poset of $\mathrm{Fl}_{\mathrm{P}}(\mathbb{F})$ is one motivation to introduce P-flag matroids (Definition [5.18), extending flag matroids. The representable ones (Definition 5.20) determine the $t_{n}$-stratification of $\mathrm{Fl}_{\mathrm{P}}(\mathbb{F})$ (Corollary [5.23). In general, we believe that a $Q$ Bruhat poset is graded, see Conjecture 5.24

The paper is organized as follows:

- In Section 2 we fix notation and we recall useful facts concerning symmetric groups, incidence algebras and matroids. Several classical topics overviewed in the section are extended in this paper.

- In Section 3 we introduce $P$-flags in a vector space (Definition 3.1). We prove that $P$-flags are parameterized by a homogeneous space $\mathrm{Fl}_{\mathrm{P}}(\mathbb{F})$ (Theorem 3.9), which for $\mathbb{F}=\mathbb{R}$ is a differentiable manifold (Corollary 3.10).

In Theorem 3.23, we describe as homogeneous spaces some of the orbits of the action of $I^{*}(Q, \mathbb{F})$ on $P$-flags, where $Q$ is any poset of cardinality $n$. This shows that also classical Schubert cells are homogeneous spaces, where the isotropy subgroups are the incidence groups of posets whose Hasse diagrams are the graphs introduced in [5, see Remark 3.20 .

- Section 4 and Section 5 are devoted to the study of incidence stratifications of Grassmannians $\operatorname{Gr}_{\mathbb{F}}(k, n)$ and $P$-flag spaces. First we provide full information about the orbits (and their Zariski closures) of the action of $I^{*}(Q, \mathbb{F})$ on $\mathbb{P}(V)$ (Theorem 4.2). Then we characterize $Q$-Schubert cells in both cases, indexing them with order ideals in suitable posets; the characterization is given in terms of representable matroids (Theorem 4.19) and sets represented by $P$-flags (Definition 5.12 and Theorem 5.15). We introduce the $Q$-Bruhat posets of $\operatorname{Gr}_{\mathbb{F}}(k, n)$ and $\mathrm{Fl}_{\mathrm{P}}(\mathbb{F})$ (Definitions 4.17 
and 5.6). The $c_{n}$-Bruhat orders coincide with the Bruhat order on $S_{n}^{(k)}$ and $S_{n}$, respectively (Propositions 4.24 and 5.16). On the other hand, the $t_{n}$-Bruhat order of $\operatorname{Gr}_{\mathbb{F}}(k, n)$ is the so-called weak order on representable matroids of rank $k$, see Remark 4.20 .

The last part of the paper describes the $c_{n}$-stratification of $\mathrm{Fl}_{t_{n}}(\mathbb{F})$ in terms of (dual) parking functions (Theorem 5.27).

\section{Notation and preliminaries}

In this section we fix notation and recall some definitions useful for the rest of the paper. We refer to [26] and [27] for posets and their incidence algebras, to 1] and [16] for the theory of Coxeter groups, to 2, 4] and [21 for matroids and flag matroids, to [7, [17, [18, and [25] for general results on Grassmannians and flag varieties.

Let $\mathbb{N}$ be the set of non-negative integers. For $n \in \mathbb{N} \backslash\{0\}$, we use the notation $[n]:=\{1,2, \ldots, n\}$. For a finite set $X \neq \varnothing$, we denote by $|X|$ its cardinality, by $\mathcal{P}(X)$ its power set, by $X^{n}$ its $n$-th power under Cartesian product and we let $X^{0}:=\{()\}$. If $x \in X^{n}$, we denote by $x_{i}$ the projection of $x$ on the $i$-th factor. The $q$-analog of $n$ is a polynomial defined by $[n]_{q}:=\sum_{i=0}^{n-1} q^{i}$; the $q$-analog of the factorial is the polynomial $[n]_{q} !:=\prod_{k \in[n]}[k]_{q}$. Let $k \in \mathbb{N}$ with $k \leqslant n$. We define the set

$$
[n]_{<}^{k}:=\left\{\left(x_{1}, \ldots, x_{k}\right) \in[n]^{k}: x_{1}<x_{2}<\ldots<x_{k}\right\} .
$$

It is clear that there exists a bijection $\bigcup_{k=0}^{n}[n]_{<}^{k} \rightarrow \mathcal{P}([n])$. Hence, the Boolean operations on $\mathcal{P}([n])$ make sense in $\bigcup_{k=0}^{n}[n]_{<}^{k}$.

The notations $\operatorname{End}(O)$ and $\operatorname{Aut}(O)$ stand for the set of endomorphisms and automorphisms of an object $O$ in a category.

The symmetric group of permutations of $n$ objects is denoted by $S_{n}$. A permutation $\sigma \in S_{n}$ can be written in one line notation as $\sigma(1) \sigma(2) \ldots \sigma(n)$. An inversion in $\sigma$ is a pair $(i, j) \in[n]_{<}^{2}$ such that $\sigma(i)>\sigma(j)$. The number of inversions in $\sigma$ is denoted by $\operatorname{inv}(\sigma)$.

For any field $\mathbb{F}$ let $\operatorname{Mat}(n, \mathbb{F})$ be the set of $n \times n$ matrices over $\mathbb{F}, \operatorname{Id}_{n}$ the identity matrix and $\operatorname{GL}(n, \mathbb{F})$ the group of invertible matrices of size $n$.

The projective space of a vector space $V$ is denoted by $\mathbb{P}(V)$ and the Grassmannians by

$$
\operatorname{Gr}_{\mathbb{F}}(k, n):=\left\{W \subseteq \mathbb{F}^{n}: W \text { is a vector subspace of dimension } k\right\} .
$$

Let $\phi: \operatorname{Gr}_{\mathbb{F}}(k, n) \rightarrow \mathbb{P}\left(\bigwedge^{k} \mathbb{F}^{n}\right)$ be the Plücker embedding, i.e. the injective function defined by $\phi(W)=\left[w_{1} \wedge \ldots \wedge w_{k}\right]$, for any basis $\left\{w_{1}, \ldots, w_{k}\right\}$ of $W \in$ $\operatorname{Gr}_{\mathbb{F}}(k, n)$.

Finally, the set of complete flags in $\mathbb{F}^{n}$ is

$$
\mathrm{Fl}_{n}(\mathbb{F}):=\left\{W_{1} \subseteq \ldots \subseteq W_{n}: W_{i} \in \operatorname{Gr}_{\mathbb{F}}(i, n), \forall i \in[n]\right\} .
$$




\subsection{Posets, incidence algebras and incidence groups}

All posets considered in this paper are finite. An interval in a poset $(X, \leqslant)$ is a subset $[x, y]:=\{z \in X: x \leqslant z \leqslant y\}$, where $x, y \in X$ and $x \leqslant y$. When $|[x, y]|=2$, we use the notation $x \triangleleft y$. The following two posets appear repeatedly in the sequel:

- $c_{n}:=([n], \leqslant)$, the chain of $n$ elements;

- $t_{n}$ the trivial poset on $[n]$, i.e. the poset without relations.

We need to introduce the following definition in order to deal with incidence algebras as matrix algebras.

Definition 2.1. Let $n>0$. Define the set of naturally labeled posets as

$$
\operatorname{POS}(n):=\left\{\left([n], \leqslant_{P}\right): i \leqslant_{P} j \Rightarrow i \leqslant j, \forall i, j \in[n]\right\} \text {. }
$$

The set of relations of $P \in \operatorname{POS}(n)$ is

$$
T_{P}:=\left\{(i, j) \in[n]_{<}^{2}: i<_{P} j\right\} .
$$

The elements of $\operatorname{POS}(n)$ can be ordered by setting

$$
P \leqslant Q \Leftrightarrow T_{P} \subseteq T_{Q}
$$

for all $P, Q \in \operatorname{POS}(n)$. This is a particular case of weak order on binary relations, as defined in 9 . The poset $(\operatorname{POS}(n), \leqslant)$ has minimum and maximum, namely $t_{n}$ and $c_{n}$, respectively.

The following notions are fundamental for the rest of this article.

Definition 2.2. The incidence algebra of a poset $P \in \operatorname{POS}(n)$ over a field $\mathbb{F}$ is

$$
I(P ; \mathbb{F}):=\left\{A \in \operatorname{Mat}(n, \mathbb{F}): A_{i, j}=0, \text { if } i>j \text { or }(i, j) \in[n]_{<}^{2} \backslash T_{P}\right\},
$$

where $A_{i, j}$ is the ij-entry of the matrix $A$. The incidence group of $P$ over $\mathbb{F}$ is

$$
I^{*}(P ; \mathbb{F}):=I(P ; \mathbb{F}) \cap \operatorname{GL}(n, \mathbb{F}) .
$$

The unipotent group of $P$ is the subgroup of $I^{*}(P ; \mathbb{F})$ defined by

$$
U(P ; \mathbb{F}):=\left\{A \in I^{*}(P ; \mathbb{F}): A_{i, i}=1, \forall i \in[n]\right\} .
$$

The algebra $I\left(t_{n} ; \mathbb{F}\right)$ is the algebra of diagonal matrices over $\mathbb{F}$. In general, it is clear that $I(P ; \mathbb{F})$ is a subalgebra of the algebra $I\left(c_{n} ; \mathbb{F}\right)$ of $n \times n$ upper triangular matrices over $\mathbb{F}$.

Notice that $P \leqslant Q$ implies $I^{*}(P ; \mathbb{F}) \subseteq I^{*}(Q ; \mathbb{F})$, for all $P, Q \in \operatorname{POS}(n)$. We are going to prove that the quotient $I^{*}(Q ; \mathbb{F}) / I^{*}(P ; \mathbb{F})$ has a nice structure, under suitable assumptions.

A graph on $n$ vertices is a pair $([n], E)$, where $E \subseteq[n]_{<}^{2}$ is the set of edges. The comparability graph of $P \in \operatorname{POS}(n)$ is the graph $\left([n], T_{P}\right)$.

Definition 2.3. Let $P, Q \in \operatorname{POS}(n)$ such that $P \leqslant Q$. We say that $P$ is complemented in $Q$ if $\left([n], T_{Q} \backslash T_{P}\right)$ is the comparability graph of a poset $P^{c}(Q)$. 
Proposition 2.4. Let $P, Q \in \operatorname{POS}(n)$. Assume $P$ complemented in $Q$. Then $U\left(P^{c}(Q) ; \mathbb{F}\right) \subseteq I^{*}(Q ; \mathbb{F})$ and we have that the canonical projection $I^{*}(Q ; \mathbb{F}) \rightarrow$ $I^{*}(Q ; \mathbb{F}) / I^{*}(P ; \mathbb{F})$ restricts to a bijection

$$
\pi_{U}: U\left(P^{c}(Q) ; \mathbb{F}\right) \rightarrow I^{*}(Q ; \mathbb{F}) / I^{*}(P ; \mathbb{F}) .
$$

Proof. If $P=Q$ then $P^{c}(Q)=t_{n}$ and the result follows. Assume $P<Q$. It is clear that $P^{c}(Q) \leqslant Q$. Then $U\left(P^{c}(Q), \mathbb{F}\right) \subseteq I^{*}(Q, \mathbb{F})$. Since $U\left(P^{c}(Q), \mathbb{F}\right) \cap$ $I^{*}(P, \mathbb{F})=\left\{\operatorname{Id}_{n}\right\}$, the function $\pi_{U}$ is injective.

It remains to prove that $\pi_{U}$ is surjective. Let $A \in I^{*}(Q ; \mathbb{F})$. It is sufficient to prove that there exists $X \in I^{*}(P ; \mathbb{F})$ with $A X \in U\left(P^{c}(Q), \mathbb{F}\right)$, because $\pi_{U}(A X)=\pi_{U}(A)$. The condition $A X \in U\left(P^{c}(Q), \mathbb{F}\right)$ is satisfied if and only if

1. $X_{i, i}=\frac{1}{A_{i, i}}$, for all $i \in[n]$ and

2. $\sum_{i \leqslant Q} \sum_{P} A_{i, k} X_{k, j}=0$, for all $(i, j) \in T_{P}$.

This gives a non homogeneous linear system whose matrix is an element of $I^{*}\left(T_{P} ; \mathbb{F}\right)$, where $T_{P}$ is the induced subposet of the Cartesian product $Q \times Q$. Then such a linear system admits a solution $X$.

Remark 2.5. The bijection of Proposition 2.4 is not a group isomorphism, because in general $I^{*}(P ; \mathbb{F})$ is not a normal subgroup of $I^{*}(Q ; \mathbb{F})$.

We end this section by defining the following duality function.

Definition 2.6. An involution $*: \operatorname{POS}(n) \rightarrow \operatorname{POS}(n)$ is defined by letting $i \leqslant_{P^{*}} j$ if and only if $n+1-j \leqslant_{P} n+1-i$, for every $P \in \operatorname{POS}(n)$. A fixed point of $*$ is called a self-dual poset.

\subsection{The symmetric groups as Coxeter groups}

A Coxeter system $(W, S)$ is a group $W$ with a presentation whose generators are the elements of a finite set $S=\left\{s_{1}, \cdots, s_{n-1}\right\}$, with relations given by $s_{i}^{2}=e$ and $\left(s_{i} s_{j}\right)^{m_{i j}}=e$, for suitable $m_{i j} \geqslant 2$ if $i \neq j$, where $e$ is the identity in $W$.

Given a Coxeter system $(W, S)$, the length function $\ell: W \rightarrow \mathbb{N}$ is defined by $\ell(w):=\min \left\{k \in \mathbb{N}: w=s_{i_{1}} s_{i_{2}} \cdots s_{i_{k}}\right\}$, for every $w \in W$.

For any $J \subseteq S$, the subgroup generated by $J$ is denoted by $W_{J}$. Define

$$
W^{J}:=\{w \in W: \ell(w s)>\ell(w), \forall s \in J\} .
$$

We recall an important result (see [1, Proposition 2.4.4]).

Proposition 2.7. Any element $w \in W$ factorizes uniquely as $w=w^{J} w_{J}$, where $w^{J} \in W^{J}, w_{J} \in W_{J}$ and $\ell(w)=\ell\left(w^{J}\right)+\ell\left(w_{J}\right)$.

Therefore one can define an idempotent function $P^{J}: W \rightarrow W$ by setting $P^{J}(w):=w^{J}$.

One of the most important features of a Coxeter group is a natural partial order $\leqslant$ on it, called Bruhat order. It can be defined by the subword property (see [1, Chapter 2] and [16, Chapter 5]). The induced subposet $\left(W^{J}, \leqslant\right)$ is graded with rank function $\ell$ (see [1, Theorem 2.5.5]) and the function $P^{J}$ is 
order preserving, i.e. $u \leqslant v$ implies $P^{J}(u) \leqslant P^{J}(v)$, for all $u, v \in W$ (see 1, Proposition 2.5.1]).

The symmetric group $S_{n}$ is a Coxeter group; its standard Coxeter presentation has generators $S=\left\{s_{1}, \ldots, s_{n-1}\right\}$, where $s_{i}$ is the permutation $12 \ldots(i+$ $1) i \ldots n$, for all $i \in[n-1]$. With respect to this presentation, $\ell(\sigma)=\operatorname{inv}(\sigma)$, for every $\sigma \in S_{n}$. Hence the element of maximal length is $w_{0}=n(n-1) \ldots 21$.

The following example should make clear how to obtain the permutation $P^{J}(\sigma)$. For more information about how $P^{J}$ rearranges a permutation, we refer to [1, Section 2.4].

Example 2.8. Let $n=7, J=\left\{s_{1}, s_{2}, s_{4}, s_{6}\right\}$ and $\sigma=4317625$. Therefore we have to rearrange increasingly the blocks 431, 76 and 25 . It follows that $P^{J}(\sigma)=1346725$.

We denote $S_{n}^{S \backslash\left\{s_{k}\right\}}$ by $S_{n}^{(k)}$, for all $k \in[n-1]$, and we set $S_{n}^{(n)}:=\{e\}$. It is clear that

$$
S_{n}^{(k)}=\left\{\sigma \in S_{n}: \sigma(1)<\cdots<\sigma(k), \sigma(k+1)<\cdots<\sigma(n)\right\},
$$

for all $k \in[n]$. The elements of $S_{n}^{(k)}$ are called Grassmannian permutations since they index the set of Schubert varieties of $\operatorname{Gr}_{\mathbb{C}}(k, n)$. Moreover the set $S_{n}^{(k)}$ is in bijection with the set $[n]_{<}^{k}$, for all $k \in[n]$. For example, $2357146 \in S_{7}^{(4)}$ corresponds to $(2,3,5,7) \in[7]_{<}^{4}$.

By the next result the Bruhat order on $S_{n}^{(k)}$ corresponds to the componentwise ordering of $[n]_{<}^{k}$ (see [1, Proposition 2.4.8]).

Proposition 2.9. The induced Bruhat order on $S_{n}^{(k)}$ is described by

$$
\sigma \leqslant \tau \text { if and only if } \sigma(i) \leqslant \tau(i) \text {, for every } i \in[k-1] \text {, }
$$

for all $\sigma, \tau \in S_{n}^{(k)}$.

By 1, Theorem 2.6.1], the Bruhat order on $S_{n}$ can be given in terms of the posets $\left(S_{n}^{(k)}, \leqslant\right), k \in[n]$. Namely $\sigma \leqslant \tau$ if and only if $P^{S \backslash\left\{s_{k}\right\}}(\sigma) \leqslant P^{S \backslash\left\{s_{k}\right\}}(\tau)$, for all $k \in[n-1]$.

The poset $\left(S_{n}^{(k)}, \leqslant\right)$ is isomorphic to the set of Schubert varieties in $\operatorname{Gr}_{\mathbb{C}}(k, n)$ ordered by inclusion. Analogously, $\left(S_{n}, \leqslant\right)$ is the poset of Schubert varieties in $\mathrm{Fl}_{n}(\mathbb{C})$ ordered by inclusion.

We are also interested in the so-called Gale ordering on $S_{n}^{(k)}$.

Definition 2.10. The Gale ordering $\leqslant^{\sigma}$ on $S_{n}^{(k)}$ induced by $\sigma \in S_{n}$, is defined by letting $u \leqslant^{\sigma} v$ if and only if $P^{S \backslash\left\{s_{k}\right\}}(\sigma u) \leqslant P^{S \backslash\left\{s_{k}\right\}}(\sigma v)$, for all $u, v \in S_{n}^{(k)}$.

For example, let $u=2413567, v=5712346$ in $S_{7}^{(2)}$, and $\sigma=3256174 \in S_{7}$. Then $u \leqslant^{e} v$. Moreover $\sigma u=2635174, \sigma v=1432567$ and $P^{S \backslash\left\{s_{2}\right\}}(\sigma v)=$ $1423567 \leqslant 2613457=P^{S \backslash\left\{s_{2}\right\}}(\sigma u)$. Therefore $v \leqslant{ }^{\sigma} u$.

Following [4, Section 1.7], we define the Gale order on a symmetric group.

Definition 2.11. The Gale ordering $\leqslant^{\sigma}$ on $S_{n}$ induced by $\sigma \in S_{n}$, is defined by letting $u \leqslant \sigma \quad v$ if and only if $\sigma u \leqslant \sigma v$, for all $u, v \in S_{n}$.

This is equivalent to require $P^{S \backslash\left\{s_{k}\right\}}(u) \leqslant{ }^{\sigma} P^{S \backslash\left\{s_{k}\right\}}(v)$, for every $k \in[n-1]$.

For example, let $u=324561, v=623541$ in $S_{6}$, and $\sigma=325614 \in S_{6}$. Then $u \leqslant^{e} v$. Moreover $\sigma u=526143, \sigma v=425163$ and $526143 \nless 425163$. Hence $u \nless^{\sigma} v$. 


\subsection{Matroids}

Let $n>0$ and $k \in[n]$. A set $M \subseteq S_{n}^{(k)}$ is a matroid ${ }^{3}$ of rank $k$ if it satisfies the Maximality Property:

the induced subposet $\left(M, \leqslant^{\sigma}\right)$ has maximum, for all $\sigma \in S_{n}$.

Remark 2.12. Since the left multiplication by $w_{0}$ is an antinvolution of the poset $\left(S_{n}, \leqslant\right)$, the Maximality Property is equivalent to saying that $\left(M, \leqslant^{\sigma}\right)$ has minimum, for all $\sigma \in S_{n}$, i.e. has maximum and minimum, for all $\sigma \in S_{n}$.

The set of matroids in $[n]_{<}^{k}$ can be ordered by inclusion (this is usually called weak order, see e.g. [32, Chapter 9]).

Let $W \in \operatorname{Gr}_{\mathbb{F}}(k, n)$ and $\left\{v_{1}, \ldots, v_{k}\right\} \subseteq \mathbb{F}^{n}$ be a basis of $W$. If $\left\{e_{1}, \ldots, e_{n}\right\}$ is the canonical basis of $\mathbb{F}^{n}$, one has that

$$
v_{1} \wedge \ldots \wedge v_{k}=\sum_{i \in[n]_{<}^{k}} a_{i} e_{i_{1}} \wedge \ldots \wedge e_{i_{k}} .
$$

It is well known that $M(W):=\left\{i \in[n]_{<}^{k}: a_{i} \neq 0\right\}$ is the set of bases of a matroid. Recall that we identify the set $S_{n}^{(k)}$ with $[n]_{<}^{k}$. We say that a matroid $M \subseteq[n]_{<}^{k}$ is representable over a field $\mathbb{F}$ if there exists a vector space $W \in$ $\operatorname{Gr}_{\mathbb{F}}(k, n)$ such that $M=M(W)$. The equivalence relation $W_{1} \sim W_{2}$ if and only if $M\left(W_{1}\right)=M\left(W_{2}\right)$, for all $W_{1}, W_{2} \in \mathrm{Gr}_{\mathbb{F}}(k, n)$, provides the matroid stratification of $\operatorname{Gr}_{\mathbb{F}}(k, n)$ introduced and studied in [14].

Remark 2.13. Notice that the equivalence classes of the relation $\sim$ are given by the intersection between $\phi\left(\operatorname{Gr}_{\mathbb{F}}(k, n)\right)$ and the orbits of the action of the group of invertible diagonal matrices, of size $\left(\begin{array}{l}n \\ k\end{array}\right)$, on $\mathbb{P}\left(\bigwedge^{k} \mathbb{F}^{n}\right)$, where $\phi$ is the Plücker embedding.

By using [3. Theorem 3.3], it is not difficult to characterize Bruhat intervals in $S_{n}^{(k)}$ as particular types of transversal matroids, namely lattice path matroids, in the meaning of [3, Definition 3.1] (see also [19, Definition 22]). By [19, Lemma 23] they are positroids.

We recall the following extension of the notion of matroid.

Definition 2.14. A subset $F \subseteq S_{n}$ such that the induced subposet $\left(F, \leqslant^{\sigma}\right)$ has maximum for all $\sigma \in S_{n}$, is said to be a flag matroid.

By [8, Theorem 4.4], any Bruhat interval in $S_{n}$ is a flag matroid. For sake of completeness we provide a proof of the following property which gives a connection between flag matroids and matroids.

Proposition 2.15. Let $k \in[n-1]$. If $F \subseteq S_{n}$ is a flag matroid, then $\left\{P^{S \backslash\left\{s_{k}\right\}}(f): f \in F\right\}$ is a matroid of rank $k$.

Proof. We set $J_{k}:=S \backslash\left\{s_{k}\right\}$. Let $\sigma \in S_{n}$ and $f_{\sigma}$ be the maximum of the poset $\left(F, \leqslant^{\sigma}\right)$. We claim that $P^{J_{k}}\left(f_{\sigma}\right)$ is the maximum of $\left\{P^{J_{k}}(f): f \in F\right\} \subseteq S_{n}^{(k)}$ with respect to $\leqslant^{\sigma}$. Let $u \in F$; then $u \leqslant{ }^{\sigma} f_{\sigma}$, i.e. $\sigma u \leqslant \sigma f_{\sigma}$. Recall that the projection $P^{J_{k}}$ is order preserving. Then $P^{J_{k}}(\sigma u) \leqslant P^{J_{k}}\left(\sigma f_{\sigma}\right)$. We have that $P^{J_{k}}(\sigma u)=P^{J_{k}}\left(\sigma u^{J_{k}} u_{J_{k}}\right)=P^{J_{k}}\left(\sigma u^{J_{k}}\right)$ and similarly $P^{J_{k}}\left(\sigma f_{\sigma}\right)=P^{J_{k}}\left(\sigma f_{\sigma}^{J_{k}}\right)$ then $P^{J_{k}}\left(\sigma P^{J_{k}}(u)\right) \leqslant P^{J_{k}}\left(\sigma P^{J_{k}}\left(f_{\sigma}\right)\right)$, i.e. $P^{J_{k}}(u) \leqslant{ }^{\sigma} P^{J_{k}}\left(f_{\sigma}\right)$. This concludes the proof.

\footnotetext{
${ }^{3}$ More precisely, the set of bases of a matroid.
} 


\section{$3 \quad$ P-flag spaces}

In this section we introduce a class of homogeneous spaces which is one the main object of our study, recovering as particular cases the flag varieties and the moduli space of $n$ independent lines in $\mathbb{C}^{n}$.

Let $\mathbb{F}$ be a field, $n>0$ and $P \in \operatorname{POS}(n)$. Consider $V:=\mathbb{F}^{n}$, the $\mathbb{F}$-vector space with canonical basis $\left\{e_{i}: i \in[n]\right\}$. Given any subset $I \subseteq[n]$, we define the vector subspace

$$
V_{I}:=\operatorname{span}_{\mathbb{F}}\left\{e_{i}: i \in I\right\} .
$$

Recall that an order ideal in a poset $P$ is a subset $I \subseteq P$ such that $i \in I$ and $j \leqslant_{P} i$ imply $j \in I$. The distributive lattice of order ideals of a poset $P \in \operatorname{POS}(n)$ is denoted by $\mathcal{J}(\mathrm{P})$. It is clear that there is a bijection between $\mathcal{J}(\mathrm{P})$ and the antichains of $P$, i.e. the set $\{\max (I): I \in \mathcal{J}(\mathrm{P})\}$.

For $i \in[n]$, we define the principal order ideal generated by $i \in P$ by setting

$$
i^{\downarrow}:=\left\{j \in[n]: j \leqslant_{P} i\right\} .
$$

Given a subset $I \subseteq[n]$, we define $I^{\downarrow}:=\bigcup_{i \in I} i^{\downarrow}$, the order ideal of $P$ generated by

$I$. We write $i_{P}^{\downarrow}$ and $I_{P}^{\downarrow}$ whenever we need to stress the poset under consideration. Notice that the number of relations of $P$ is $\left|T_{P}\right|=\sum_{i \in[n]}\left|i^{\downarrow}\right|-n$.

The following is one of the main definition of this article.

Definition 3.1. A $P$-flag in $V$ is an $n$-tuple $\left(V_{1}, \ldots, V_{n}\right)$ of vector subspaces of $V$ which satisfies the following condition:

$$
\operatorname{dim}\left(\sum_{i \in I} V_{i}\right)=\left|I^{\downarrow}\right|,
$$

for every $I \subseteq[n]$. The set of $P$-flags of $V$ is denoted by $\mathrm{Fl}_{\mathrm{P}}(\mathbb{F})$.

We call standard P-flag of $V$ the tuple

$$
F_{e}^{P}:=\left(V_{1^{\downarrow}}, \ldots, V_{n^{\downarrow}}\right) .
$$

A $c_{n}$-flag is a complete flag in the usual meaning. On the other hand, a $t_{n}$-flag is an $n$-tuple of lines in $\mathbb{F}^{n}$ whose generators are linearly independent. The following example shows an intermediate case between the previous ones.

Example 3.2. Let $V=\mathbb{F}^{6}$ and $P \in \operatorname{POS}(6)$ be the poset in the figure below:

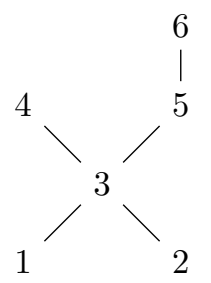

Let us consider the following vector subspaces of $V$ :

- $W_{1}:=\operatorname{span}_{\mathbb{F}}\left\{e_{1}\right\}, W_{2}:=\operatorname{span}_{\mathbb{F}}\left\{e_{2}\right\}$, 
- $W_{3}:=\operatorname{span}_{\mathbb{F}}\left\{e_{1}, e_{2}, e_{3}\right\}, W_{4}:=\operatorname{span}_{\mathbb{F}}\left\{e_{1}, e_{2}, e_{3}, e_{4}\right\}$,

- $W_{5}:=\operatorname{span}_{\mathbb{F}}\left\{e_{1}, e_{2}, e_{3}, e_{5}\right\}, W_{6}:=\operatorname{span}_{\mathbb{F}}\left\{e_{1}, e_{2}, e_{3}, e_{5}, e_{6}\right\}$.

Then $\left(W_{1}, W_{2}, W_{3}, W_{4}, W_{5}, W_{6}\right)$ is the standard P-flag.

The tuples $\left(W_{1}, W_{2}, W_{3}, W_{4}, W_{6}, W_{5}\right)$ and $\left(W_{1}, W_{1}, W_{3}, W_{4}, W_{5}, W_{6}\right)$ are not $P$-flags. Examples of $P$-flags are

$$
\left(W_{1}, W_{2}, W_{3}, W_{5}, W_{4}, W_{6}\right) \text { and }\left(W_{2}, W_{1}, W_{3}, W_{5}, W_{4}, W_{6}\right) \text {. }
$$

Recall that for $F \in \mathrm{Fl}_{\mathrm{P}}(\mathbb{F}), F_{i}$ is the projection on the $i$-th factor. The following proposition states some properties of a $P$-flag.

Proposition 3.3. Let $F \in \mathrm{Fl}_{\mathrm{P}}(\mathbb{F})$. Then

1. $\operatorname{dim}\left(F_{i}\right)=\left|i^{\downarrow}\right|$, for all $i \in[n]$;

2. $\operatorname{dim}\left(F_{i} \cap F_{j}\right)=\left|i^{\downarrow} \cap j^{\downarrow}\right|$;

3. $F_{i} \subseteq F_{j}$ if and only if $i \leqslant_{P} j$;

4. $\sum_{i \in[n]} F_{i}=V$.

Proof. Properties 1. and 4. are obtained by Definition 3.1, taking $I=\{i\}$ and $I=[n]$, respectively.

By the Grassmann formula and Property 1., $\operatorname{dim}\left(F_{i} \cap F_{j}\right)=\operatorname{dim}\left(F_{i}\right)+$ $\operatorname{dim}\left(F_{j}\right)-\operatorname{dim}\left(F_{i}+F_{j}\right)=\left|i^{\downarrow}\right|+\left|j^{\downarrow}\right|-\left|i^{\downarrow} \cup j^{\downarrow}\right|=\left|i^{\downarrow} \cap j^{\downarrow}\right|$.

To prove Property 3., let $F_{i} \subseteq F_{j}$. This holds if and only if $\operatorname{dim}\left(F_{i} \cap F_{j}\right)=$ $\operatorname{dim}\left(F_{i}\right)$. But this is equivalent to $\left|i^{\downarrow} \cap j^{\downarrow}\right|=\left|i^{\downarrow}\right|$, which is equivalent to $i^{\downarrow} \subseteq j^{\downarrow}$, i.e. $i \leqslant P j$.

Remark 3.4. Let $F \in \mathrm{Fl}_{\mathrm{P}}(\mathbb{F})$. Note that, by Property 3. of Proposition 3.3 , $F_{i}=F_{j}$ if and only if $i=j$.

Remark 3.5. Let $F:=\left(W_{1}, \ldots, W_{n}\right) \in \mathrm{Fl}_{\mathrm{P}}(\mathbb{F})$ and $\sigma \in S_{n}$ such that $\sigma F:=$ $\left(W_{\sigma^{-1}(1)}, \ldots, W_{\sigma^{-1}(n)}\right) \in \mathrm{Fl}_{\mathrm{P}}(\mathbb{F})$. Then $\sigma^{-1}(1) \prec \ldots \prec \sigma^{-1}(n)$ is a linear extension of $P$. In fact let $i<_{P} j, \sigma(i)=: h$ and $\sigma(j)=: k$. Then, by Proposition 3.3. $W_{i} \subseteq W_{j}$. Since $(\sigma F)_{h}=W_{i}$ and $(\sigma F)_{k}=W_{j}$, we have that $h \leqslant_{P} k$ and this implies $h<k$, so $\sigma^{-1}(h) \prec \sigma^{-1}(k)$. It is straightforward to check that in general the converse does not hold.

We are going to prove that the set of $P$-flags admits a structure of homogeneous space. To do this, we need the following function.

Definition 3.6. The Fon-Der-Flaass action (see [24]) is the invertible function $\Psi_{P}: \mathcal{J}(\mathrm{P}) \rightarrow \mathcal{J}(\mathrm{P})$ defined by

$$
\Psi_{P}(I):=\left[\min _{P}([n] \backslash I)\right]^{\downarrow},
$$

for all $I \in \mathcal{J}(\mathrm{P})$.

Notice that $\Psi_{P}(\varnothing)=\min (P)$ and $\Psi_{P}(P)=\varnothing$. Now we are ready to prove one of the main results of this section. 
Proposition 3.7. Let $F \in \mathrm{Fl}_{\mathrm{P}}(\mathbb{F})$. Then there exists a basis $B:=\left\{v_{1}, \ldots, v_{n}\right\}$ of $V$ such that $F_{i} \cap B=\left\{v_{j} \in B: j \in i^{\downarrow}\right\}$, for all $i \in[n]$.

Proof. For any $k \geqslant 1$, define the induced subposet

$$
P_{k}:=\bigcup_{j=1}^{k} \Psi_{P}^{j}(\varnothing)
$$

and consider the vector space $W_{k}:=\sum_{i \in \max \left(P_{k}\right)} F_{i}$. It is clear that there exists $k \in \mathbb{N}$ such that $P_{k}=P$. If $P_{k}=\left\{i_{1}, \cdots, i_{\left|P_{k}\right|}\right\}$, being $i_{1}<\ldots<i_{\left|P_{k}\right|}$, then $\left(F_{i_{1}}, \ldots, F_{i_{\left|P_{k}\right|}}\right) \in \mathrm{Fl}_{P_{k}}(\mathbb{F})$, since the order ideals of $P_{k}$ are order ideals of $P$.

We construct the basis $B$ by induction on $k$. Let $k=1$. Then $P_{1}=\min (P)$ and $F_{i}=\operatorname{span}_{\mathbb{F}}\left\{v_{i}\right\}$ for some $v_{i} \in V$, for all $i \in \min (P)$. Since $\operatorname{dim}\left(\sum_{i \in P_{1}} F_{i}\right)=$ $\left|P_{1}\right|$, we have that $\left|\left\{v_{i}: i \in P_{1}\right\}\right|=\left|P_{1}\right|$ and the elements $v_{1}, \ldots, v_{\left|P_{1}\right|}$ are linearly independent. We let $B^{1}:=\left\{v_{i}: i \in P_{1}\right\}$. Then $F_{i} \cap B^{1}=\left\{v_{i}\right\}=$ $\left\{v_{j} \in B^{1}: j \in i^{\downarrow}\right\}$, for all $i \in P_{1}$.

Now let $k>1$. By induction, we have a basis $B^{k-1}$ of $W_{k-1}$ such that $F_{i} \cap B^{k-1}=\left\{v_{j} \in B^{k-1}: j \in i^{\downarrow}\right\}$, for all $i \in P_{k-1}=P_{k} \backslash \max \left(P_{k}\right)$.

Let $\max \left(P_{k}\right)=\left\{p_{1}, \ldots, p_{r}\right\}$; by Proposition 3.3, $F_{q} \subseteq F_{p_{i}}$ for all $q \triangleleft p_{i}$, $i \in[r]$, and

$$
\operatorname{dim}\left(\sum_{q \triangleleft p_{i}} F_{q}\right)=\left|\left\{q \in P_{k}: q \triangleleft p_{i}\right\}^{\downarrow}\right|=\left|p_{i}^{\downarrow}\right|-1=\operatorname{dim}\left(F_{p_{i}}\right)-1 .
$$

This implies the existence of an element $v_{p_{i}} \in F_{p_{i}} \backslash\left(\sum_{q \triangleleft p_{i}} F_{q}\right)$, for all $i \in[r]$. We let

$$
B^{k}:=B^{k-1} \cup\left\{v_{p_{1}}, \ldots, v_{p_{r}}\right\} .
$$

It remains to prove that $B^{k}$ is a basis of $W_{k}$. Let $i \in[r]$ and assume by contradiction $v_{p_{i}} \in \sum_{j \in P_{k} \backslash\left\{p_{i}\right\}} F_{j}$. Then $F_{p_{i}} \subseteq \sum_{j \in P_{k} \backslash\left\{p_{i}\right\}} F_{j}$. Hence

$$
\left|P_{k}\right|=\operatorname{dim}\left(\sum_{j \in P_{k}} F_{j}\right)=\operatorname{dim}\left(\sum_{j \in P_{k} \backslash\left\{p_{i}\right\}} F_{j}\right)=\left|P_{k}\right|-1 .
$$

If $P_{k}=P$, we let $B:=B^{k}$. Then $B$ is a basis of $V$ with the stated property.

Corollary 3.8. Let $F \in \mathrm{Fl}_{\mathrm{P}}(\mathbb{F})$. Then the set $\left\{F_{i}: i \in[n]\right\}$ generates, by sums and intersections, a distributive lattice isomorphic to $\mathcal{J}(P)$. Moreover

$$
\operatorname{dim}\left(\bigcap_{i \in I} F_{i}\right)=\left|\bigcap_{i \in I} i^{\downarrow}\right|
$$

for all $I \subseteq[n]$. 
Proof. By Proposition 3.7 the lattice generated by $\left\{F_{i}: i \in[n]\right\}$ is isomorphic to the lattice $L$ generated by $\left\{F_{i} \cap B: i \in[n]\right\}$, with respect to the operations $\cup$ and $\cap$, which is distributive. From this we deduce also the last assertion. Moreover, by construction $L$ is isomorphic to $\mathcal{J}(P)$.

Let $F \in \mathrm{Fl}_{\mathrm{P}}(\mathbb{F})$. If $B:=\left\{v_{1}, \ldots, v_{n}\right\}$ is a basis of $V$ such that $F_{i} \cap B=$ $\left\{v_{j} \in B: j \in i^{\downarrow}\right\}$, for all $i \in[n]$, we say that $B$ is $F$-adapted. Choosing an $F$-adapted basis $\left\{v_{1}, \ldots, v_{n}\right\}$ of $V$ for any $P$-flag $F \in \mathrm{Fl}_{\mathrm{P}}(\mathbb{F})$, we can define a function $\beta: \mathrm{Fl}_{\mathrm{P}}(\mathbb{F}) \rightarrow \mathrm{GL}(n, \mathbb{F})$ by setting $\beta(F)$ as the unique matrix which satisfies $\beta(F) e_{i}=v_{i}$, for all $i \in[n]$.

Theorem 3.9. Let $\pi: \operatorname{GL}(n, \mathbb{F}) \rightarrow \mathrm{GL}(n, \mathbb{F}) / I^{*}(P ; \mathbb{F})$ be the canonical projection. Then the function

$$
\pi \circ \beta: \mathrm{Fl}_{\mathrm{P}}(\mathbb{F}) \rightarrow \mathrm{GL}(n, \mathbb{F}) / I^{*}(P ; \mathbb{F})
$$

is bijective.

Proof. An action of the group $\mathrm{GL}(n, \mathbb{F})$ on $\mathrm{Fl}_{\mathrm{P}}(\mathbb{F})$ is given by

$$
(A F)_{i}:=A F_{i},
$$

for all $i \in[n], A \in \mathrm{GL}(n, \mathbb{F})$, and $F \in \mathrm{Fl}_{\mathrm{P}}(\mathbb{F})$. In fact dimensions are preserved and $A\left(\sum_{i \in I} F_{i}\right)=\sum_{i \in I} A F_{i}$ for all $I \subseteq[n], A \in \operatorname{GL}(n, \mathbb{F})$. Since $\beta(F) F_{e}^{P}=F$, for all $F \in \mathrm{Fl}_{\mathrm{P}}(\mathbb{F})$, this action is transitive and $A F_{e}^{P}=F_{e}^{P}$ if and only $A \in I^{*}(P ; \mathbb{F})$, so the result follows.

For arbitrary fields, we call $\mathrm{Fl}_{\mathrm{P}}(\mathbb{F})$ a $P$-flag space. The set $\mathrm{Fl}_{\mathrm{P}}(\mathbb{R})$ turns out to have a structure of differentiable manifold, which we call $P$-flag manifold. We recover the real flag manifold for $P=c_{n}$.

Corollary 3.10. Let $P \in \operatorname{POS}(n)$. The set $\mathrm{Fl}_{\mathrm{P}}(\mathbb{R})$ is a differentiable manifold of dimension $n(n-1)-\left|T_{P}\right|$.

Proof. Notice that $I^{*}(P ; \mathbb{R})$ is a closed subgroup of the Lie group $\mathrm{GL}(n ; \mathbb{R})$; in fact an incidence group is defined by the vanishing of suitable entries, depending on $P$. By the closed-subgroup theorem (see, e.g. [15, Theorem 9.3.7]), $I^{*}(P ; \mathbb{R})$ is a Lie subgroup and, by [15, Theorem 10.1.10], the quotient $\mathrm{GL}(n, \mathbb{R}) / I^{*}(P ; \mathbb{R})$ has a unique real manifold structure.

Since the Lie algebra of $I^{*}(P ; \mathbb{R})$ is the Lie algebra of the incidence algebra $I(P ; \mathbb{R})$ and its dimension is $|P|+\left|T_{P}\right|$, we obtain the stated formula (see, e.g. [15, Corollary 10.1.12]).

Remark 3.11. By Theorem 3.9, there exists a canonical projection $\mathrm{Fl}_{\mathrm{P}}(\mathbb{C}) \rightarrow$ $\mathrm{Fl}_{n}(\mathbb{C})$ whose fibers are affine spaces of dimension $\left|[n]_{<}^{2} \backslash T_{P}\right|$. It follows that this projection is a homotopy equivalence.

By Theorem 3.9 we can deduce the cardinality of the set of $P$-flags on a finite field of $q$ elements.

Corollary 3.12. Let $P \in \operatorname{POS}(n)$. Then

$$
\left|\mathrm{Fl}_{\mathrm{P}}\left(\mathbb{F}_{q}\right)\right|=q^{\frac{n(n-1)}{2}-\left|T_{P}\right|}[n]_{q} !
$$


Proof. First of all recall the well-known formula

$$
\left|\operatorname{GL}\left(n ; \mathbb{F}_{q}\right)\right|=\prod_{i=0}^{n-1}\left(q^{n}-q^{i}\right)=q^{\frac{n(n-1)}{2}} \prod_{i=1}^{n}\left(q^{i}-1\right) .
$$

It is clear that $\left|I^{*}\left(P ; \mathbb{F}_{q}\right)\right|=(q-1)^{n} q^{\left|T_{P}\right|}$. Then the result follows from Theorem 3.9 .

The following proposition reveals a duality phenomenon, which does not appear in the classical case, since a chain $c_{n}$ is self-dual (see Definition [2.6).

Proposition 3.13. Let $P \in \operatorname{POS}(n)$. Then we have a bijection

$$
\mathrm{Fl}_{P}^{*}: \mathrm{Fl}_{\mathrm{P}}(\mathbb{F}) \rightarrow \mathrm{Fl}_{\mathrm{P}^{*}}(\mathbb{F})
$$

defined by setting

$$
\mathrm{Fl}_{P}^{*}(F)_{i}=\operatorname{span}_{\mathbb{F}}\left\{v_{n+1-j}: j \in i_{P^{*}}^{\downarrow}\right\}
$$

for all $i \in[n], F \in \mathrm{Fl}_{\mathrm{P}}(\mathbb{F})$, where $\left\{v_{1}, \ldots, v_{n}\right\}$ is an $F$-adapted basis of $V$.

Proof. Let $F \in \mathrm{Fl}_{\mathrm{P}}(\mathbb{F})$ and $\left\{v_{1}, \ldots, v_{n}\right\}$ be an $F$-adapted basis of $V$. Let $w_{i}:=$ $v_{n+1-i}$, for all $i \in[n]$; therefore, by Definition [2.6. $\left\{w_{1}, \ldots, w_{n}\right\}$ is an $\mathrm{Fl}^{*}(F)$ adapted basis of $V$. It is clear by construction that $\mathrm{Fl}_{P^{*}}^{*} \circ \mathrm{Fl}_{P}^{*}$ and $\mathrm{Fl}_{P}^{*} \circ \mathrm{Fl}_{P^{*}}^{*}$ are the identity on $\mathrm{Fl}_{\mathrm{P}}(\mathbb{F})$ and $\mathrm{Fl}_{\mathrm{P} *}(\mathbb{F})$, respectively.

In the example below we present in a particular case the duality in Proposition 3.13

Example 3.14. Given a positive integer $n$, the $n$-th configuration space of a set $X$ is

$$
\operatorname{Conf}_{n}[X]:=\left\{\left(x_{1}, \ldots, x_{n}\right) \in X^{n}: i \neq j \Rightarrow x_{i} \neq x_{j}\right\} .
$$

Unless otherwise specified, the symbol $\simeq$ stands for a bijection.

Let $P \in \operatorname{POS}(3)$ be the poset whose Hasse diagram is the one on the left in the following figure. The Hasse diagram on the right is the one of $P^{*}$.
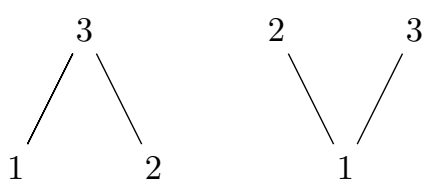

Let $\left\{v_{1}, v_{2}, v_{3}\right\}$ be a basis of $V, V_{1}:=\operatorname{span}_{\mathbb{F}}\left\{v_{1}\right\}, V_{2}:=\operatorname{span}_{\mathbb{F}}\left\{v_{2}\right\}$ and $V_{3}:=V$. Then $F:=\left(V_{1}, V_{2}, V_{3}\right) \in \mathrm{Fl}_{\mathrm{P}}(\mathbb{F})$ and

$$
\mathrm{Fl}_{P}^{*}(F)=\left(\operatorname{span}_{\mathbb{F}}\left\{v_{3}\right\}, \operatorname{span}_{\mathbb{F}}\left\{v_{2}, v_{3}\right\}, \operatorname{span}_{\mathbb{F}}\left\{v_{1}, v_{3}\right\}\right) .
$$

Moreover, it is immediate to check that $\mathrm{Fl}_{\mathrm{P}}(\mathbb{F}) \simeq \operatorname{Conf}_{2}\left[\mathbb{P}\left(\mathbb{F}^{3}\right)\right]$ and $\mathrm{Fl}_{\mathrm{P}}(\mathbb{F}) \simeq$ $\operatorname{Conf}_{2}\left[\operatorname{Gr}_{\mathbb{F}}(2,3)\right] \simeq \operatorname{Conf}_{2}\left[\mathbb{P}\left(\mathbb{F}^{3}\right)\right]$.

Remark 3.15. We observe that $\mathrm{Fl}_{\mathrm{P}}(\mathbb{C})$ is a subset of the moduli space of spanning configurations $X_{\alpha, n}$, introduced in [23], with $\alpha=\left(\left|1^{\downarrow}\right|, \ldots,\left|n^{\downarrow}\right|\right)$. Moreover $\mathrm{Fl}_{t_{n}}(\mathbb{C})=X_{1^{n}, n}$, where $1^{n}=(1,1, \ldots, 1) \in[1]^{n}$. Notice that $\mathrm{Fl}_{t_{n}}(\mathbb{C})$ is also the moduli space $X_{n, n}$ of $n$ independent lines in $\mathbb{C}^{n}$ of [22]. 


\section{$3.1(Q, P)$-cells}

In this section we consider the left action of the incidence group $I^{*}(Q ; \mathbb{F})$ on $\operatorname{Fl}_{\mathrm{P}}(\mathbb{F})$, where $P, Q \in \operatorname{POS}(n)$. For $P=Q=c_{n}$, the orbits of this action are the classical Schubert cells of the flag variety, which are indexed by the elements of the symmetric group $S_{n}$. In Proposition 3.16 we prove that for other choices of $Q$ and $P$, the action of $I^{*}(Q ; \mathbb{R})$ on $\mathrm{Fl}_{\mathrm{P}}(\mathbb{R})$ has infinitely many orbits. For other general results on infiniteness of double quotients see for instance [10, [13] and references therein. Nevertheless we consider a finite subset of these orbits, corresponding to permutations in $S_{n}$, which have a particularly nice description as in the classical case.

Proposition 3.16. The double quotient $I^{*}(Q ; \mathbb{R}) \backslash \mathrm{GL}(n ; \mathbb{R}) / I^{*}(P ; \mathbb{R})$ is finite if and only if $P=Q=c_{n}$.

Proof. It is well known that if $P=Q=c_{n}$ then the double quotient considered is in bijection with the symmetric group $S_{n}$.

Let $Q$ be any poset and $P \neq c_{n}$. The maximal possible dimension $d$ of an orbit of $I^{*}(Q ; \mathbb{R})$ is reached when $Q=c_{n}$ and the isotropy group is the group of invertible diagonal matrices $I^{*}\left(t_{n} ; \mathbb{F}\right) ;$ then $d=\operatorname{dim}\left(I^{*}\left(c_{n} ; \mathbb{R}\right)\right)-n=\frac{n(n-1)}{2}$ by [15, Corollary 10.1.12]. By Corollary $3.10, \operatorname{dim}\left(\operatorname{GL}(n ; \mathbb{R}) / I^{*}(P ; \mathbb{R})\right)=n(n-$ $1)-\left|T_{P}\right|$. Since $P \neq c_{n}$, the minimum of $n(n-1)-\left|T_{P}\right|$ is reached when $P$ has exactly two incomparable elements; its value is $n(n-1)-\frac{n(n-1)-2}{2}=\frac{n(n-1)}{2}+1$. Therefore $\operatorname{dim}\left(\mathrm{GL}(n ; \mathbb{R}) / I^{*}(P ; \mathbb{R})\right)$ is always strictly greater than the dimension of every orbit of $I^{*}(Q ; \mathbb{R})$, which implies the infiniteness of the set of such orbits.

Now we consider a collection of orbits of $I^{*}(Q ; \mathbb{F})$ on $\mathrm{Fl}_{\mathrm{P}}(\mathbb{F})$ which share some properties with the classical Schubert cells of the flag variety. For any permutation $\sigma \in S_{n}$, let us define the $P$-flag

$$
F_{\sigma}^{P}:=\left(\operatorname{span}_{\mathbb{F}}\left\{e_{\sigma(i)}: i \in 1_{P}^{\downarrow}\right\}, \ldots, \operatorname{span}_{\mathbb{F}}\left\{e_{\sigma(i)}: i \in n_{P}^{\downarrow}\right\}\right) .
$$

When $\sigma$ is the identity we recover the standard $P$-flag $F_{e}^{P}$.

Definition 3.17. The $(Q, P)$-cell in $\mathrm{Fl}_{\mathrm{P}}(\mathbb{F})$ corresponding to $\sigma \in S_{n}$ is the orbit

$$
C_{\sigma}^{Q, P}(\mathbb{F}):=\left\{A F_{\sigma}^{P}: A \in I^{*}(Q ; \mathbb{F})\right\} .
$$

These cells can be described as homogeneous spaces. Before to state this result, we need some definitions.

Definition 3.18. Let $P, Q \in \operatorname{POS}(n)$ and $\sigma \in S_{n}$. The poset $[Q P]_{\sigma}:=$ $([n], \leqslant Q, P, \sigma)$ is defined by setting

$$
i \leqslant_{Q, P, \sigma} j \Leftrightarrow i \leqslant_{Q} j \text { and } \sigma^{-1}(i) \leqslant_{P} \sigma^{-1}(j)
$$

for every $i, j \in[n]$.

Notice that $[Q P]_{\sigma} \leqslant Q$, for every $P, Q \in \operatorname{POS}(n), \sigma \in S_{n}$.

Example 3.19. Let $Q \in \operatorname{POS}(n)$. It is clear that $\left[Q c_{n}\right]_{e}=Q$ and $\left[Q t_{n}\right]_{\sigma}=t_{n}$ for all $\sigma \in S_{n}$. Moreover $\left[Q c_{n}\right]_{w_{0}}=t_{n}$, where $w_{0}=n \cdots 321$. 
Remark 3.20. The Hasse diagram of the poset $\left[c_{n} c_{n}\right]_{\sigma}$ is the graph $G_{\sigma}$ defined in [5]. This is also related to the inversion graph of the permutation $\sigma$ (see [20]).

Remark 3.21. The induced subposet $\left\{\left[c_{n} c_{n}\right]_{\sigma}: \sigma \in S_{n}\right\} \subseteq \operatorname{POS}(n)$ is isomorphic to the dual of the right $\leqslant_{R}$ weak order of $S_{n}$. In fact, by [1, Proposition 3.1.3], $\sigma \leqslant_{R} \tau$ if and only if $T_{L}(\sigma) \subseteq T_{L}(\tau)$, where $T_{L}(\sigma)$ is the set of left inversions of $\sigma$. This is equivalent to $\left[c_{n} c_{n}\right]_{\tau} \leqslant\left[c_{n} c_{n}\right]_{\sigma}$.

Definition 3.22. Let $\sigma \in S_{n}$. The $(Q, P)$-inversion number $\operatorname{inv}_{Q, P}(\sigma)$ of $\sigma$ is defined by

$$
\operatorname{inv}_{Q, P}(\sigma):=\left|\left\{(i, j) \in[n]_{<}^{2}: i<_{Q} j, \sigma^{-1}(i) \nless_{P} \sigma^{-1}(j)\right\}\right| .
$$

For $Q=P=c_{n}$ this function gives the usual inversion number $\operatorname{inv}(\sigma)$ of a permutation in $S_{n}$.

Theorem 3.23. Let $P, Q \in \operatorname{POS}(n)$ and $\sigma \in S_{n}$. Then we have the following bijections:

$$
C_{\sigma}^{Q, P}(\mathbb{F}) \simeq I^{*}(Q ; \mathbb{F}) / I^{*}\left([Q P]_{\sigma} ; \mathbb{F}\right) \simeq \mathbb{F}^{\operatorname{inv}_{Q, P}(\sigma)} .
$$

Proof. Let $F_{\sigma}^{P}=\left(V_{1}, \ldots, V_{n}\right)$, where $V_{j}=\operatorname{span}_{\mathbb{F}}\left\{e_{\sigma(i)}: i \in j_{P}^{\downarrow}\right\}$, for all $j \in[n]$. Let $A \in I^{*}(Q ; \mathbb{F})$ be an element of the isotropy group of $F_{\sigma}^{P}$ under the action $A F_{\sigma}^{P}=\left(A V_{1}, \ldots, A V_{n}\right)$. We prove that $A \in I^{*}\left([Q P]_{\sigma} ; \mathbb{F}\right)$.

We have that $V_{1}=\operatorname{span}_{\mathbb{F}}\left\{e_{\sigma(1)}\right\}$ and $A V_{1}=V_{1}$ implies $A_{i, \sigma(1)}=0$ for all $i<_{Q} \sigma(1)$. Again $A V_{2}=V_{2}$ implies $A_{i, \sigma(2)}=0$ for all $i<_{Q} \sigma(2)$ such that $i \notin\left\{\sigma(k): k \in 2_{P}^{\downarrow}\right\}$. In general, $A V_{j}=V_{j}$ implies $A_{i, \sigma(j)}=0$ for all $i<_{Q} \sigma(j)$ such that $i \notin\left\{\sigma(k): k \in j_{P}^{\downarrow}\right\}$. Therefore the isotropy group of $F_{\sigma}^{P}$ is contained in the set

$$
\begin{gathered}
\bigcap_{j=1}^{n}\left\{A \in I^{*}(Q ; \mathbb{F}): A_{i, \sigma(j)}=0, \forall i \notin\left\{\sigma(k): k \in j_{P}^{\downarrow}\right\}\right\}= \\
\bigcap_{j=1}^{n}\left\{A \in I^{*}(Q ; \mathbb{F}): A_{i, j}=0, \forall i \notin\left\{k: \sigma^{-1}(k) \in\left[\sigma^{-1}(j)\right]_{P}^{\downarrow}\right\}\right\}=I^{*}\left([Q P]_{\sigma} ; \mathbb{F}\right) .
\end{gathered}
$$

By definition of $F_{\sigma}^{P}$ and $[Q P]_{\sigma}$, it follows that $I^{*}\left([Q P]_{\sigma} ; \mathbb{F}\right)$ is contained in the isotropy group of $F_{\sigma}^{P}$, and the first bijection is proved.

A coset of $A \in I^{*}(Q ; \mathbb{F})$ is determined setting $A_{i, i}=1$ for all $i \in[n]$ and $A_{i j}=0$ whenever $(i, j) \in T_{[Q P]_{\sigma}}$. Since $\left|T_{Q} \backslash T_{[Q P]_{\sigma}}\right|=\operatorname{inv}_{Q, P}(\sigma)$, the second bijection follows.

Immediate consequences of Theorem 3.23 are the following statements.

Corollary 3.24. Let $\mathbb{F}_{q}$ be a finite field. Then

$$
\left|C_{\sigma}^{Q, P}\left(\mathbb{F}_{q}\right)\right|=q^{\operatorname{inv}_{Q, P}(\sigma)},
$$

for all $\sigma \in S_{n}$.

A poset is said to be strict Sperner if it is a graded poset in which all maximum antichains are rank levels. The next result gives a bijection between a $(Q, P)$-cell $C_{\sigma}^{Q, P}(\mathbb{F})$ and the derived algebra of the Lie algebra $I\left([Q P]_{\sigma}^{c}(Q) ; \mathbb{F}\right)$, whenever $P$ is a strict Sperner poset. 
Corollary 3.25. If $P$ is strict Sperner, then we have a bijection

$$
C_{\sigma}^{Q, P}(\mathbb{F}) \simeq U\left([Q P]_{\sigma}^{c}(Q) ; \mathbb{F}\right),
$$

for all $\sigma \in S_{n}$.

Proof. By definition, if $P$ is strict Sperner then the poset $[Q P]_{\sigma}$ is complemented in $Q$. In fact, in a strict Sperner poset, the relation $\nless$ is transitive. Then $T_{Q} \backslash T_{[Q P]_{\sigma}}=T_{[Q P]_{\sigma}(Q)}$. Hence the result follows by Proposition 2.4

\section{Incidence stratifications}

In this section we provide a partition of the projective space $\mathbb{P}\left(\mathbb{F}^{n}\right)$, induced by the action of the incidence group $I^{*}(Q ; \mathbb{F})$, for any poset $Q \in \operatorname{POS}(n)$. The orbits of such an action turn out to be in one-to-one correspondence with the elements of the distributive lattice $\mathcal{J}(\mathrm{Q})$.

This decomposition induces a partition of any subset of a projective space. We investigate the induced partition on Grassmannian varieties, recovering the Schubert cell partition, for $Q=c_{n}$, and the matroid strata introduced in [14, for $Q=t_{n}$.

\subsection{Q-stratification of a projective space}

Let $Q \in \operatorname{POS}(n), V=\mathbb{F}^{n}$ and $\mathbb{P}(V)$ its projective space. The subalgebra $I(Q ; \mathbb{F}) \subseteq \operatorname{End}(V)$ has invariant-subspace lattice isomorphic to $\mathcal{J}(Q)$, where $I(Q ; \mathbb{F})$ acts on the elements of $V$ by left multiplication.

Remark 4.1. The socle filtration of the action of $I(Q ; \mathbb{F})$ on $V$ is given by

$$
\operatorname{soc}^{i}(Q) \simeq \bigoplus_{j \in \max \left[\Psi_{Q}^{i}(\varnothing)\right]} \operatorname{span}_{\mathbb{F}}\left\{e_{j}\right\},
$$

for all $i>0$ such that $\Psi_{Q}^{i}(\varnothing) \subsetneq \Psi_{Q}^{i+1}(\varnothing)$, where $\Psi_{Q}$ is the function of Definition 3.6.

Clearly this action carries an action of $I^{*}(Q, \mathbb{F})$ on $\mathbb{P}(V)$, whose orbits are described in the following theorem. Recall that $V_{I}:=\operatorname{span}_{\mathbb{F}}\left\{e_{i}: i \in I\right\}$, for any subset $I \subseteq[n]$.

Theorem 4.2. An orbit of the action of $I^{*}(Q ; \mathbb{F})$ on $\mathbb{P}(V)$ is of the form

$$
Q_{I}(\mathbb{F}):=\mathbb{P}\left(V_{I}\right) \backslash \bigcup_{i \in \max (I)} \mathbb{P}\left(V_{I \backslash\{i\}}\right),
$$

for any $I \in \mathcal{J}(\mathrm{Q}) \backslash\{\varnothing\}$ and the collection of cell $\left\{\sqrt{4}\left\{Q_{I}(\mathbb{F}): I \in \mathcal{J}(\mathrm{Q}) \backslash\{\varnothing\}\right\}\right.$ is a partition of $\mathbb{P}(V)$. The Zariski closure of $Q_{I}(\mathbb{C})$ is given by

$$
\overline{Q_{I}(\mathbb{C})}=\biguplus_{H \in \mathcal{J}(I) \backslash\{\varnothing\}} Q_{H}(\mathbb{C})=\mathbb{P}\left(V_{I}\right),
$$

for all $I \in \mathcal{J}(\mathrm{Q})$.

\footnotetext{
${ }^{4}$ The use of the word cell in this article does not refer in general to affine spaces.
} 
Proof. Let $v \in V$ be expressed as $v=a_{1} e_{i_{1}}+\ldots+a_{k} e_{i_{k}}$ for some $k \in[n]$, $a_{1}, \ldots, a_{k} \in \mathbb{F} \backslash\{0\}$. Let $M:=\max _{Q}\left\{i_{1}, \ldots, i_{k}\right\}$ and $I:=M^{\downarrow} \in \mathcal{J}(\mathrm{Q})$. Then $v$ lies in $V_{I} \backslash \bigcup_{i \in M} V_{I \backslash\{i\}}$. Since the action of $I^{*}(Q ; \mathbb{F})$ on $V_{I} \backslash \bigcup_{i \in M} V_{I \backslash\{i\}}$ is transitive and the projection of this set on $\mathbb{P}(V)$ is $\mathbb{P}\left(V_{I}\right) \backslash \bigcup_{i \in M} \mathbb{P}\left(V_{I \backslash\{i\}}\right)$, the first assertion follows. Finally we have that $\overline{Q_{I}(\mathbb{C})}=\mathbb{P}\left(V_{I}\right)$; since $V_{I}$ is $I^{*}(Q ; \mathbb{C})$-invariant, the last assertion can be deduced by repeating the previous arguments to the projective space $\mathbb{P}\left(V_{I}\right)$.

In analogy with the case $\mathbb{F}=\mathbb{C}$ in Theorem 4.2, for any field $\mathbb{F}$, we say that $Q_{I}(\mathbb{F})$ is a $Q$-Schubert cell of $\mathbb{P}(V)$ and we define $\overline{Q_{I}(\mathbb{F})}:=\bigcup_{H \in \mathcal{J}(I) \backslash\{\varnothing\}} Q_{H}(\mathbb{F})$, saying that $\overline{Q_{I}(\mathbb{F})}$ is a $Q$-Schubert variety of $\mathbb{P}(V)$, which turns out to be a projective space.

The following are immediate consequences of Theorem 4.2

Corollary 4.3. Let $Q \in \operatorname{POS}(n)$ and $I \in \mathcal{J}(\mathrm{Q}) \backslash\{\varnothing\}$. Then

$$
\left.\operatorname{dim}\left(\overline{Q_{I}(\mathbb{C}}\right)\right)=|I|-1 .
$$

Corollary 4.4. The poset of $Q$-Schubert varieties of $\mathbb{P}(V)$, ordered by inclusion, is isomorphic to $\mathcal{J}(\mathrm{Q}) \backslash\{\varnothing\}$. Moreover, if $I \cap J \neq \varnothing$ then

$$
\overline{Q_{I}(\mathbb{F})} \cap \overline{Q_{J}(\mathbb{F})}=\overline{Q_{I \cap J}(\mathbb{F})} .
$$

In the case of a finite field $\mathbb{F}_{q}$, we provide a formula for the number of points of a $Q$-Schubert cell $Q_{I}\left(\mathbb{F}_{q}\right)$.

Corollary 4.5. Let $\mathbb{F}_{q}$ be a finite field. Then

$$
\left|Q_{I}\left(\mathbb{F}_{q}\right)\right|=\sum_{\substack{H \in \mathcal{J}(I) \backslash\{\varnothing\} \\ I \backslash H \subseteq \max (I)}}(-1)^{|I \backslash H|}[|H|]_{q} .
$$

Proof. By Theorem 4.2 we know that $\mathbb{P}\left(V_{I}\right)=\underset{H \in \mathcal{J}(I) \backslash\{\varnothing\}}{\biguplus} Q_{H}\left(\mathbb{F}_{q}\right)$. It is known (see [27, Example 3.9.6]) that the Möbius function of a distributive lattice is

$$
\mu(H, I)= \begin{cases}(-1)^{|I \backslash H|}, & \text { if } I \backslash H \subseteq \max (I) ; \\ 0, & \text { otherwise. }\end{cases}
$$

Since $\left|\mathbb{P}\left(\mathbb{F}_{q}^{n}\right)\right|=[n]_{q}$, we obtain our formula by Möbius inversion.

With the following definition we introduce a general procedure to decompose subsets of projective spaces. In the subsequent sections we apply this approach to Grassmannians and $P$-flag spaces.

Definition 4.6. Let $X \subseteq \mathbb{P}\left(\mathbb{F}^{n}\right)$. Given a poset $Q \in \operatorname{POS}(n)$, we call incidence stratification of $X$ the set

$$
\left\{\overline{Q_{I}(\mathbb{F})} \cap X: I \in \mathcal{J}(Q)\right\} \backslash\{\varnothing\} .
$$




\subsection{Q-stratification of a Grassmannian}

Let $n>0, k \in[n]$ and $Q \in \operatorname{POS}(n)$. We need to define a suitable poset $Q_{<}^{k}$ in order to realize an incidence stratification of the $\operatorname{Grassmannian}_{\mathrm{Gr}_{\mathbb{F}}}(k, n)$, generalizing Schubert varieties and matroidal strata.

Consider the Cartesian $k$-th power $Q^{k}$ of the poset $Q$. Recall that the order on $Q^{k}$ is defined by

$$
i \leqslant_{Q^{k}} j \Leftrightarrow i_{h} \leqslant_{Q} j_{h}, \text { for every } h \in[k],
$$

for all $i, j \in[n]^{k}$, where $i_{h}$ is the projection of $i$ on the $h$-th component.

The poset $Q^{k}$ admits an action of the symmetric group $S_{k}$, as showed in the next proposition, whose proof is straightforward.

Proposition 4.7. Let $\sigma \in S_{k}$. Then the action on $[n]^{k}$ defined by

$$
\sigma i:=\left(i_{\sigma^{-1}(1)}, \ldots, i_{\sigma^{-1}(k)}\right),
$$

for all $i \in[n]^{k}$, is an automorphism of the poset $Q^{k}$. This defines a group morphism $S_{k} \rightarrow \operatorname{Aut}\left(Q^{k}\right)$.

The following poset is fundamental for our constructions.

Definition 4.8. The poset $Q_{<}^{k}:=\left([n]_{<}^{k}, \preccurlyeq_{Q^{k}}\right)$ is defined by letting

$$
i \preccurlyeq Q^{k} j \Leftrightarrow \sigma i \leqslant_{Q^{k}} j,
$$

for some $\sigma \in S_{k}$, for all $i, j \in[n]_{<}^{k}$.

Notice that $Q_{<}^{1}=Q$. For $k>1$ it could be not obvious that $Q_{<}^{k}$ is a poset. This follows from Proposition 4.7, as we are going to show. Let $i, j, h \in[n]_{<}^{k}$.

1. reflexivity: straightforward, by taking $\sigma=e$.

2. antisymmetry: let $\sigma i \leqslant_{Q^{k}} j$ and $\tau j \leqslant_{Q^{k}} i$, for some $\sigma, \tau \in S_{k}$. Then $\tau \sigma i \leqslant_{Q^{k}} i$. From the fact that $i_{1}<\ldots<i_{k}$, we obtain $\tau \sigma=e$. Hence $i \leqslant_{Q^{k}} \tau j \leqslant_{Q^{k}} i$, which implies $\tau=\sigma=e$ and $i=j$.

3. transitivity: let $h \preccurlyeq_{Q^{k}} i$ and $i \preccurlyeq_{Q^{k}} j$; then there exist $\sigma, \tau \in S_{k}$ such that $\sigma h \leqslant_{Q^{k}} i \leqslant_{Q^{k}} \tau j$. This implies $\tau^{-1} \sigma h \leqslant_{Q^{k}} j$.

It is clear that $i \leqslant_{Q^{k}} j$ implies $i \preccurlyeq Q_{Q^{k}} j$, i.e. the poset $Q_{<}^{k}$ is a refinement of $\left([n]_{<}^{k}, \leqslant_{Q^{k}}\right)$, the induced subposet of $Q^{k}$. If $Q=c_{n}$, they are actually the same poset, as stated in the following proposition.

Proposition 4.9. Let $n \geqslant 1$ and $k \in[n]$. Then $\left(c_{n}\right)_{<}^{k}=\left([n]_{<}^{k}, \leqslant_{c_{n}^{k}}\right)$.

Proof. Let $i, j \in[n]_{<}^{k}$ with $i \Varangle_{c_{n}^{k}} j$. Then there exists a minimal $h \in[k]$ such that $j_{h}<i_{h}$. If $h=k$ then it is immediate to check that $\sigma i \Varangle_{c_{n}^{k}} j$, for all $\sigma \in S_{k}$. Let $h<k$ and $\sigma \in S_{k}$. There are three cases to be considered.

1. $\sigma^{-1}(h)=h$ : we have that $j_{h}<i_{h}=i_{\sigma^{-1}(h)}$ and this implies $\sigma i \Varangle_{c_{n}^{k}} j$.

2. $\sigma^{-1}(h)>h$ : in this case $i_{\sigma^{-1}(h)}>i_{h}>j_{h}$, so $\sigma i \Varangle_{c_{n}^{k}} j$.

3. $\sigma^{-1}(h)<h$ : in this case $h>1$. There exists $t \in[h-1]$ such that $\sigma^{-1}(t) \geqslant h$; then $i_{\sigma^{-1}(t)} \geqslant i_{h}>j_{h}>j_{t}$ and $\sigma i \Varangle_{c_{n}^{k}} j$. 
Then $i \preccurlyeq c_{n}^{k} j$ implies $i \leqslant_{c_{n}^{k}} j$.

We can consider $Q_{<}^{k}$ as an element of POS $\left(\left(\begin{array}{l}n \\ k\end{array}\right)\right)$; in fact the lexicographic order on $[n]_{<}^{k}$ provides a natural labeling of $Q_{<}^{k}$, as showed in the next proposition.

Proposition 4.10. Let $Q \in \operatorname{POS}(n)$ and $k \geqslant 1$. Then $a \preccurlyeq Q_{Q^{k}} b \Rightarrow a \leqslant_{\operatorname{lex}} b$, for all $a, b \in[n]_{<}^{k}$.

Proof. We claim that $Q \leqslant P$ implies $Q_{<}^{k} \hookrightarrow P_{<}^{k}$, for all $Q, P \in \operatorname{POS}(n)$. In fact, $\sigma a \leqslant{Q^{k}} b$ implies $\sigma a \leqslant P^{k} b$, for all $a, b \in[n]_{<}^{k}, \sigma \in S_{k}$. Since $Q \leqslant c_{n}$, we obtain $Q_{<}^{k} \hookrightarrow\left(c_{n}\right)_{<}^{k}$. By Proposition 4.9, $\left(c_{n}\right)_{<}^{k}=\left([n]_{<}^{k}, \preccurlyeq c_{n}^{k}\right)=\left([n]_{<}^{k}, \leqslant_{c_{n}^{k}}\right)$. Moreover we have that $\left([n]_{<}^{k}, \leqslant_{c_{n}^{k}}\right) \hookrightarrow\left([n]_{<}^{k}, \leqslant_{\text {lex }}\right)$ is a linear extension of $\left(c_{n}\right)_{<}^{k}$. Then $Q_{<}^{k} \hookrightarrow\left(c_{n}\right)_{<}^{k} \hookrightarrow\left([n]_{<}^{k}, \leqslant\right.$ lex $) \simeq c_{\left(\begin{array}{c}n \\ k\end{array}\right)}$ gives a linear extension of $Q_{<}^{k}$.

The duality proved in the following proposition is a poset theoretic version of the Grassmannian duality $\operatorname{Gr}_{\mathbb{F}}(k, n) \simeq \operatorname{Gr}_{\mathbb{F}}(n-k, n)$.

Proposition 4.11. Let $Q \in \operatorname{POS}(n)$. Then the following poset isomorphism holds for all $k \in[n-1]$ :

$$
Q_{<}^{k} \simeq\left(Q_{<}^{n-k}\right)^{*} .
$$

Proof. Let $a, b \in[n]_{<}^{k}$. Recall that we consider $\bigcup_{k=0}^{n}[n]_{<}^{k}$ as the Boolean algebra $\mathcal{P}([n])$. We let $g^{c}:=[n] \backslash g \in[n]_{<}^{n-k}$, for all $g \in[n]_{<}^{k}$. We claim that $a \preccurlyeq Q^{k} b$ if and only if $a \backslash b \preccurlyeq Q^{h} b \backslash a$, where $h:=k-m$ and $m:=|a \cap b|$. If $a \cap b=\varnothing$ there is nothing to prove. Assume $a \cap b \neq \varnothing$.

1. $a \preccurlyeq Q_{Q^{k}} b \Rightarrow a \backslash b \preccurlyeq Q_{Q^{h}} b \backslash a$ : by hypothesis there exists $\omega \in S_{k}$ such that $a \leqslant_{Q^{k}} \omega b$. Let $a_{i}:=z \in a \cap b, a_{j}:=x \leqslant_{Q} z=:(\omega b)_{j}$ and $z \leqslant_{Q} y=:(\omega b)_{i}$. Then $x \leqslant_{Q} y$ and $a \leqslant_{Q^{k}}(\tau \omega) b$, where, if $i \neq j, \tau \in S_{k}$ is the transposition such that $(\tau \omega b)_{j}=y$ and $(\tau \omega b)_{i}=z$, otherwise $\tau$ is the identity. We then conclude by repeated use of this argument.

2. $a \backslash b \preccurlyeq Q^{h} b \backslash a \Rightarrow a \preccurlyeq Q_{Q^{k}} \quad b$ : by hypothesis there exists $\omega \in S_{h}$ such that $a \backslash b \leqslant_{Q^{h}} \omega(b \backslash a)$. Let $\sigma, \tau \in S_{k}$ be the permutations such that $\sigma a=\left(u_{1}, \ldots, u_{m}, v_{1}, \ldots, v_{h}\right)$ and $\tau b=\left(u_{1}, \ldots, u_{m}, z_{1}, \ldots, z_{h}\right)$, where $\left(u_{1}, \ldots, u_{m}\right)=a \cap b,\left(v_{1}, \ldots, v_{h}\right)=a \backslash b$ and $\left(z_{1}, \ldots, z_{h}\right)=\omega(b \backslash a)$. Hence $\sigma a \leqslant_{Q^{k}} \tau b$ and this implies $a \preccurlyeq Q_{Q^{k}} b$.

Notice that $a^{c} \backslash b^{c}=b \backslash a$ and $b^{c} \backslash a^{c}=a \backslash b$; hence, by the previous claim we have that

$$
\begin{aligned}
a \preccurlyeq Q_{Q^{k}} b & \Leftrightarrow a \backslash b \preccurlyeq Q_{Q^{h}} b \backslash a \\
& \Leftrightarrow \quad b^{c} \backslash a^{c} \preccurlyeq Q^{h} a^{c} \backslash b^{c} \\
& \Leftrightarrow b^{c} \preccurlyeq Q^{n-k} a^{c},
\end{aligned}
$$

where $h:=k-|a \cap b|$.

Remark 4.12. By the proof of Proposition 4.11. we know that $a \preccurlyeq Q_{Q^{k}} b$ if and only if $a \backslash b \preccurlyeq Q^{h} b \backslash a$, for all $a, b \in[n]_{<}^{k}$, where $h:=k-|a \cap b|$. This is very useful when dealing with explicit examples of the poset $Q_{<}^{k}$. 
Let $Q \in \operatorname{POS}(n)$; there exists a representation $\pi_{Q}^{k}: I^{*}(Q ; \mathbb{F}) \rightarrow \operatorname{Aut}\left(\bigwedge^{k} V\right)$ given by diagonal action:

$$
A\left(v_{1} \wedge \ldots \wedge v_{k}\right)=A v_{1} \wedge \ldots \wedge A v_{k}
$$

for every $A \in I^{*}(Q ; \mathbb{F})$ and $v_{1}, \ldots, v_{k} \in V$.

Theorem 4.13. The group morphism $\pi_{Q}^{k}$ is injective and $\pi_{Q}^{k}\left(I^{*}(Q ; \mathbb{F})\right)$ is a subgroup of the incidence group $I^{*}\left(Q_{<}^{k} ; \mathbb{F}\right)$.

Proof. Let $A \in I^{*}(Q ; \mathbb{F})$ such that $\pi_{Q}^{k}(A)=\mathrm{Id}$. Then any subspace of dimension $k$ of $V$ is $A$-invariant. This implies that $A=\operatorname{Id}_{n}$. Moreover we have that, for $i \in Q_{<}^{k}$,

$$
\begin{aligned}
A e_{i_{1}} \wedge \ldots \wedge A e_{i_{k}}= & \left(\sum_{h \in i_{1}^{\downarrow}} A_{h, i_{1}} e_{h}\right) \wedge \ldots \wedge\left(\sum_{h \in i_{k}^{\downarrow}} A_{h, i_{k}} e_{h}\right) \\
& \in \bigoplus_{j \in I} \operatorname{span}_{\mathbb{F}}\left\{e_{j_{1}} \wedge \ldots \wedge e_{j_{k}}\right\},
\end{aligned}
$$

where $I:=\left\{j \in[n]_{<}^{k}: j \preccurlyeq Q_{Q^{k}} i\right\}$.

Let $\phi: \operatorname{Gr}_{\mathbb{F}}(k, n) \rightarrow \mathbb{P}\left(\bigwedge^{k} V\right)$ be the Plücker embedding. According to the action of the incidence group $I^{*}\left(Q_{<}^{k} ; \mathbb{F}\right)$ on $\mathbb{P}\left(\bigwedge^{k} V\right)$, we provide an incidence stratification of the Grassmannian $\operatorname{Gr}_{\mathbb{F}}(k, n)$.

Definition 4.14. Let $Q_{I}(\mathbb{F})$ be an orbit of the action of $I^{*}\left(Q_{<}^{k} ; \mathbb{F}\right)$ on the projective space $\mathbb{P}\left(\bigwedge^{k} V\right)$, for any order ideal $I \in \mathcal{J}\left(Q_{<}^{k}\right)$. The set

$$
[Q]_{I}(\mathbb{F}):=\left(Q_{<}^{k}\right)_{I}(\mathbb{F}) \cap \phi\left(\operatorname{Gr}_{\mathbb{F}}(k, n)\right)
$$

is called $Q$-Schubert cell of $\mathrm{Gr}_{\mathbb{F}}(k, n)$ whenever $[Q]_{I}(\mathbb{F}) \neq \varnothing$. A $Q$-Schubert variety in $\operatorname{Gr}_{\mathbb{F}}(k, n)$ is $\overline{[Q]_{I}(\mathbb{F})}:=\overline{\left(Q_{<}^{k}\right)_{I}(\mathbb{F})} \cap \phi\left(\operatorname{Gr}_{\mathbb{F}}(n, k)\right)$.

The next result follows directly from Definition 4.14 and Theorem 4.2 .

Proposition 4.15. Let $I \in \mathcal{J}\left(Q_{<}^{k}\right)$ and $[Q]_{I}(\mathbb{F})$ be a Q-Schubert cell. We have that

$$
\overline{[Q]_{I}(\mathbb{F})}=\biguplus_{H \in \mathcal{J}(I)}[Q]_{H}(\mathbb{F}) .
$$

Now we are going to prove that, in a Grassmannian variety, a $c_{n}$-Schubert cell is a Schubert cell. In other words, a Schubert cell is the intersection of $\operatorname{Gr}_{\mathbb{F}}(k, n)$ with a $\left(c_{n}\right)_{<}^{k}$-Schubert cell of the projective space $\mathbb{P}\left(\bigwedge^{k} V\right)$.

Proposition 4.16. Let $\sigma \in S_{n}^{(k)}$ be a Grassmannian permutation and $C_{\sigma}(\mathbb{F})$ the corresponding Schubert cell of $\operatorname{Gr}_{\mathbb{F}}(k, n)$. Then

$$
C_{\sigma}(\mathbb{F})=\left[c_{n}\right]_{I_{\sigma}}(\mathbb{F}),
$$

where $I_{\sigma}:=\left\{a \in[n]_{<}^{k}: a \preccurlyeq c_{n}^{k}(\sigma(1), \ldots, \sigma(k))\right\}$. 
Proof. A Schubert cell $C_{\sigma}(\mathbb{F})$ in $\phi\left(\operatorname{Gr}_{\mathbb{F}}(k, n)\right)$ is an orbit under the action of $\pi_{c_{n}}^{k}\left(I^{*}\left(c_{n} ; \mathbb{F}\right)\right)$ of the line $\operatorname{span}_{\mathbb{F}}\left\{e_{\sigma(1)} \wedge \ldots \wedge e_{\sigma(k)}\right\}$. By Theorem 4.13, the group $\pi_{c_{n}}^{k}\left(I^{*}\left(c_{n} ; \mathbb{F}\right)\right)$ is a subgroup of $I^{*}\left(\left(c_{n}\right)_{<}^{k} ; \mathbb{F}\right)$; therefore the orbits of the action of $I^{*}\left(\left(c_{n}\right)_{<}^{k} ; \mathbb{F}\right)$ on $\mathbb{P}\left(\bigwedge^{k} V\right)$ are partitioned into orbits of $\pi_{c_{n}}^{k}\left(I^{*}\left(c_{n} ; \mathbb{F}\right)\right)$. But the Schubert cells give a partition of $\operatorname{Gr}_{\mathbb{F}}(k, n)$, so the result follows.

By the fact that a Schubert variety is union of Schubert cells according to the Bruhat order of $S_{n}^{(k)}$, the $c_{n}$-Schubert varieties in $\operatorname{Gr}_{\mathbb{C}}(k, n)$ are exactly the Schubert varieties.

We define the set of $Q$-Schubert cells of $\mathrm{Gr}_{\mathbb{F}}(k, n)$ as

$$
Q_{B}^{k}(\mathbb{F}):=\left\{I \in \mathcal{J}\left(Q_{<}^{k}\right):[Q]_{I}(\mathbb{F}) \neq \varnothing\right\} .
$$

Definition 4.17. Let $Q \in \operatorname{POS}(n)$. We call $\left(Q_{B}^{k}(\mathbb{F}), \subseteq\right)$ the $Q$-Bruhat poset of $\operatorname{Gr}_{\mathbb{F}}(k, n)$.

Remark 4.18. By Proposition 4.16, the $c_{n}$-Bruhat poset of $\mathrm{Gr}_{\mathbb{F}}(k, n)$ is isomorphic to $S_{n}^{(k)}$ with the Bruhat order, which by Proposition 2.9 is isomorphic to $\left([n]_{<}^{k}, \leqslant_{c_{n}^{k}}\right)$. Moreover, by Proposition [4.9, these posets are isomorphic to $\left(c_{n}\right)_{<}^{k}$.

Notice that the poset $\left(Q_{B}^{1}(\mathbb{F}), \subseteq\right)$ is equal to $(\mathcal{J}(Q) \backslash\{\varnothing\}, \subseteq)$, for all $Q \in$ $\operatorname{POS}(n)$; see Theorem 4.2 .

We provide a characterization of the $Q$-Schubert cells in terms of matroids representable over $\mathbb{F}$.

Theorem 4.19. Let $Q \in \operatorname{POS}(n), k \in[n]$ and $I \in \mathcal{J}\left(Q_{<}^{k}\right)$. Then $[Q]_{I}(\mathbb{F}) \neq \varnothing$ if and only if $\max (I) \cup I^{\prime}$ is a matroid representable over $\mathbb{F}$, for some subset $I^{\prime} \subseteq I$.

Proof. Let $I^{\prime} \subseteq I$ be any subset. The result follows by observing that $\max (I) \cup I^{\prime}$ is the set of bases of a matroid representable over $\mathbb{F}$ if and only if there exists $A \in I^{*}\left(Q_{<}^{k} ; \mathbb{F}\right)$ such that

$$
A\left(\sum_{i \in \max (I)} e_{i_{1}} \wedge \ldots \wedge e_{i_{k}}\right)=\sum_{i \in \max (I) \cup I^{\prime}} a_{i} e_{i_{1}} \wedge \ldots \wedge e_{i_{k}}
$$

is an element of $\phi\left(\operatorname{Gr}_{\mathbb{F}}(k, n)\right)$, where $a_{i} \in \mathbb{F} \backslash\{0\}$ for all $i \in \max (I)$.

Remark 4.20. By Theorem $4.19,\left(\left(t_{n}\right)_{B}^{k}(\mathbb{F}), \subseteq\right)$ is the poset of representable matroids on $\mathbb{F}$ of rank $k$ on the set $[n]$, ordered by inclusion of the sets of bases. This is the so called weak order on matroids, see e.g. [21, Chapter 7] and [32, Chapter 9].

Remark 4.21. It follows by basic topology that the Zariski closure of the orbit corresponding to a matroid $M$ in the matroid stratification of $\operatorname{Gr}_{\mathbb{C}}(k, n)$ is included in $\overline{\left[t_{n}\right]_{M}(\mathbb{C})}$. This inclusion can be strict as in [12, Counterexample 2.6]. Notice that the defining ideal of the $t_{n}$-Schubert variety $\overline{\left[t_{n}\right]_{M}(\mathbb{C})}$ in $\operatorname{Gr}_{\mathbb{F}}(k, n)$ is the Grassmannian ideal $P_{M}$ of the matroid $M$, as defined in [6, Section 3].

From the fact that a singleton $\left\{\left(i_{1}, \ldots, i_{k}\right)\right\}$ is always the set of bases of a matroid representable over any field, we deduce the following corollary. 
Corollary 4.22. If $I \in \mathcal{J}\left(Q_{<}^{k}\right)$ is a principal order ideal, then $[Q]_{I}(\mathbb{F}) \neq \varnothing$.

The poset $\left(Q_{B}^{k}(\mathbb{F}), \subseteq\right)$ has maximum $[n]_{<}^{k}$, the uniform matroid; it is not difficult to see that its minimal elements, which correspond to the minima of the poset $Q_{<}^{k}$, are the Grassmannian permutations $\sigma$ such that the $Q$-inversion number $\operatorname{inv}_{Q}(\sigma):=\left\{(i, j) \in[n]_{<}^{2}: \sigma(j)<_{Q} \sigma(i)\right\}$ is zero.

Example 4.23. Let $Q \in \operatorname{POS}(4)$ be the poset on [4] such that $1 \triangleleft 2,1 \triangleleft 3$, $2 \triangleleft 4$ and $3 \triangleleft 4$. Then $Q_{<}^{2}$ is the following poset:

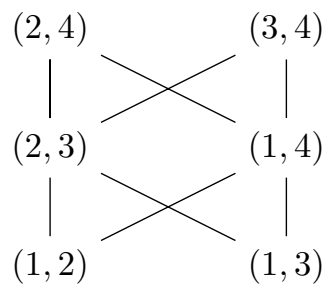

Let $\left(S_{4},\left\{s_{1}, s_{2}, s_{3}\right\}\right)$ be the symmetric group of order 24 with its standard Coxeter presentation and $J:=\left\{s_{1}, s_{3}\right\}$. The $Q$-Bruhat on $\operatorname{Gr}_{\mathbb{C}}(2,4)$ is then:

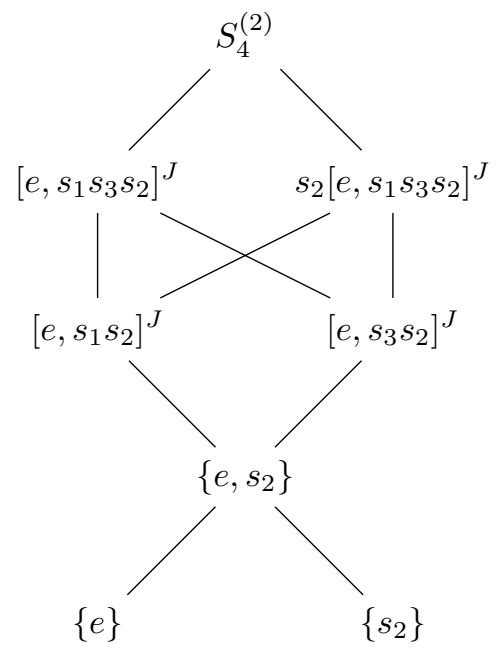

where, if $u, v \in S_{n}^{J}$ and $u \leqslant v$, then $[u, v]^{J}:=\left\{z \in S_{n}^{J}: u \leqslant z \leqslant v\right\}$ is a Bruhat interval in the poset $\left(S_{n}^{J}, \leqslant\right)$ and $w[u, v]^{J}:=\left\{P^{J}(w z): z \in[u, v]^{J}\right\}$, for all $w \in$ $S_{n}$.

By using the identification of $[4]_{<}^{2}$ with $S_{4}^{(2)}$, we have

- $\{e\}=(1,2)^{\downarrow},\left\{s_{2}\right\}=(1,3)^{\downarrow}$ and $\left\{e, s_{2}\right\}=\{(1,2),(1,3)\}^{\downarrow}$;

- $\left[e, s_{1} s_{2}\right]^{J}=(2,3)^{\downarrow}$ and $\left[e, s_{3} s_{2}\right]^{J}=(1,4)^{\downarrow}$;

- $\left[e, s_{1} s_{3} s_{2}\right]^{J}=(2,4)^{\downarrow}$ and $s_{2}\left[e, s_{1} s_{3} s_{2}\right]^{J}=(3,4)^{\downarrow}$.

Notice that, by Theorem 4.19, the order ideal $\{(2,3),(1,4)\}^{\downarrow}$ is not an element of the $Q$-Bruhat poset, i.e. $[Q]_{I}(\mathbb{F})=\varnothing$. 
By Corollary 4.22, $\left|Q_{B}^{k}(\mathbb{F})\right| \geqslant\left|S_{n}^{(k)}\right|$. In the next proposition we obtain directly that the Bruhat order on $S_{n}^{(k)}$ is the $c_{n}$-Bruhat poset, without using Proposition 4.16

Proposition 4.24. Let $n>0$ and $k \in[n]$. Then $\left(S_{n}^{(k)}, \leqslant\right) \simeq\left(\left(c_{n}\right)_{B}^{k}(\mathbb{F}), \subseteq\right)$.

Proof. Let $I_{1}, I_{2} \in \mathcal{J}\left(\left(c_{n}\right)_{<}^{k}\right)$ such $I_{1} \subseteq I_{2}$ and $\max \left(I_{1}\right) \cup I_{1}^{\prime}, \max \left(I_{2}\right) \cup I_{2}^{\prime}$ are matroids representable over $\mathbb{F}$ for some subsets $I_{1}^{\prime} \subseteq I_{1}, I_{2}^{\prime} \subseteq I_{2}$. Since the Gale order $\leqslant^{e}$ on $[n]_{<}^{k}$ is $\preccurlyeq c^{k}$, by the Maximality Property of matroids, $\left|\max \left[\max \left(I_{1}\right) \cup I_{1}^{\prime}\right]\right|=\left|\max \left(I_{1}\right)\right| \stackrel{=}{=} 1$ and $\left|\max \left[\max \left(I_{2}\right) \cup I_{2}^{\prime}\right]\right|=\left|\max \left(I_{2}\right)\right|=1$.

Moreover, if $\max (I) \in\left(c_{n}\right)_{<}^{k} \simeq S_{n}^{(k)}$ and $|\max (I)|=1$, then $\max (I)$ is clearly a representable matroid. Hence, by Theorem 4.19, $I \in\left(c_{n}\right)_{B}^{k}(\mathbb{F})$.

It is natural to go on with further investigations on the $Q$-Bruhat orders introduced in this section. For instance, supported by several computational examples, we formulate a conjecture.

Conjecture 4.25. Let $Q \in \operatorname{POS}(n)$ and $k \in[n]$. Then the poset $\left(Q_{B}^{k}(\mathbb{C}), \subseteq\right)$ is graded with rank function $\rho(I)=\operatorname{dim}\left([Q]_{I}(\mathbb{C})\right)$, for all $I \in Q_{B}^{k}(\mathbb{C})$.

Conjecture 4.25 holds when $Q=c_{n}$, since the Bruhat order on the quotients is graded with rank function the inversion number of the permutation. For $k=1$ the conjecture holds for every poset $Q$, by Corollary 4.3 . The dimension of the $t_{n}$-Schubert cells in $\operatorname{Gr}_{\mathbb{C}}(k, n)$ is provided by [31, Theorem 2.5].

\section{Incidence stratifications of P-flag spaces}

In this section we study incidence stratifications of $\mathrm{Fl}_{\mathrm{P}}(\mathbb{F})$, for every field $\mathbb{F}$. In order to do this we embed $\mathrm{Fl}_{\mathrm{P}}(\mathbb{F})$ in a projective space and we need to construct suitable posets.

Recall that $V=\operatorname{span}_{\mathbb{F}}\left\{e_{i}: i \in[n]\right\}$. Let $P \in \operatorname{POS}(n)$ and consider the function

$$
\phi_{P}: \mathrm{Fl}_{\mathrm{P}}(\mathbb{F}) \rightarrow \mathbb{P}\left(\bigotimes_{i=1}^{n} \bigwedge^{\left|i_{P}^{\downarrow}\right|} V\right),
$$

induced by the assignment

$$
F \mapsto\left(v_{1}^{1} \wedge \ldots \wedge v_{|1 \downarrow|}^{1}\right) \otimes \ldots \otimes\left(v_{1}^{n} \wedge \ldots \wedge v_{|n \downarrow|}^{n}\right),
$$

for all $F \in \operatorname{Fl}_{\mathrm{P}}(\mathbb{F})$, where $\left\{v_{1}^{i}, \ldots, v_{\mid i \downarrow}^{i}\right\}$ is any basis of $F_{i}$, for all $i \in[n]$. It is easy to see that this function is injective.

Let $Q \in \operatorname{POS}(n)$. There exists a representation

$$
\pi_{Q}: I^{*}(Q ; \mathbb{F}) \rightarrow \operatorname{Aut}\left(\bigotimes_{i=1}^{n} \bigwedge_{\left|i_{P}^{\perp}\right|} V\right)
$$

obtained extending the action of $I^{*}(Q ; \mathbb{F})$ on $V$ :

$$
A\left(v_{1}^{1} \otimes\left(v_{1}^{2} \wedge \ldots \wedge v_{|2 \downarrow|}^{2}\right) \otimes \ldots \otimes\left(v_{1}^{n} \wedge \ldots \wedge v_{|n \downarrow|}^{n}\right)\right)
$$




$$
=\left(A v_{1}^{1}\right) \otimes\left(A v_{1}^{2} \wedge \ldots \wedge A v_{\left|2^{\downarrow}\right|}^{2}\right) \otimes \ldots \otimes\left(A v_{1}^{n} \wedge \ldots \wedge A v_{\mid n \downarrow}^{n}\right),
$$

for all $A \in I^{*}(Q ; \mathbb{F})$.

Definition 5.1. Let $Q, P \in \operatorname{POS}(n)$. We define a poset

$$
Q^{P}:=Q \times Q_{<}^{\left|2_{P}^{\downarrow}\right|} \times \ldots \times Q_{<}^{\left|n_{P}^{\downarrow}\right|} .
$$

By Proposition 4.10, it is clear that the lexicographic order on $Q^{P}$ provide a natural labeling and then we consider $Q^{P} \in \operatorname{POS}\left(\left|Q^{P}\right|\right)$.

Theorem 5.2. The group morphism $\pi_{Q}$ is injective and $\pi_{Q}\left(I^{*}(Q ; \mathbb{F})\right)$ is a subgroup of the incidence group $I^{*}\left(Q^{P} ; \mathbb{F}\right)$.

Proof. Let $A \in I^{*}(Q ; \mathbb{F})$ be such that $\pi_{Q}(A)=\mathrm{Id}$. Then $A v_{1} \in \operatorname{span}_{\mathbb{F}}\left\{v_{1}\right\}$ for all $v_{1} \in V$, i.e. $A$ is the identity matrix. The other assertion follows by Theorem 4.13.

We can decompose the projective space $\mathbb{P}\left(\bigotimes_{i=1}^{n} \bigwedge^{\left|i_{P}^{\downarrow}\right|} V\right)$ according to the action of the incidence group $I^{*}\left(Q^{P} ; \mathbb{F}\right)$, giving an incidence stratification of $\operatorname{Fl}_{\mathrm{P}}(\mathbb{F})$.

Definition 5.3. Let $Q_{I}^{P}(\mathbb{F})$ be an orbit of the action of $I^{*}\left(Q^{P} ; \mathbb{F}\right)$ on the projective space $\mathbb{P}\left(\bigotimes_{i=1}^{n} \wedge^{\left|i^{\downarrow}\right|} V\right)$, for any order ideal $I \in \mathcal{J}\left(Q^{P}\right)$. The set

$$
[Q]_{I}^{P}(\mathbb{F}):=Q_{I}^{P}(\mathbb{F}) \cap \phi_{P}\left(\mathrm{Fl}_{\mathrm{P}}(\mathbb{F})\right)
$$

is called $Q$-Schubert cell of $\mathrm{Fl}_{\mathrm{P}}(\mathbb{F})$, whenever $[Q]_{I}^{P}(\mathbb{F}) \neq \varnothing . \quad A Q$-Schubert variety in $\mathrm{Fl}_{\mathrm{P}}(\mathbb{F})$ is defined by $\overline{[Q]_{I}^{P}(\mathbb{F})}:=\overline{Q_{I}^{P}(\mathbb{F})} \cap \phi_{P}\left(\mathrm{Fl}_{\mathrm{P}}(\mathbb{F})\right)$.

The next result follows directly from Definition 5.3 and Theorem 4.2 .

Proposition 5.4. Let $I \in \mathcal{J}\left(Q^{P}\right)$ and $[Q]_{I}^{P}(\mathbb{F})$ be a $Q$-Schubert cell of $\mathrm{Fl}_{\mathrm{P}}(\mathbb{F})$. We have that

$$
\overline{[Q]_{I}^{P}(\mathbb{F})}=\biguplus_{H \in \mathcal{J}(I)}[Q]_{H}^{P}(\mathbb{F}) .
$$

The following proposition asserts that, in a flag variety, a $c_{n}$-Schubert cell is a Schubert cell. In other words, a Schubert cell is the intersection of $\mathrm{Fl}_{n}(\mathbb{F})$ with a $\left(c_{n}\right)^{c_{n}}$-cell of the projective space $\mathbb{P}\left(\bigotimes_{i=1}^{n} \bigwedge^{i} V\right)$.

Proposition 5.5. Let $\sigma \in S_{n}$ and $C_{\sigma}(\mathbb{F})$ be the corresponding Schubert cell of $\mathrm{Fl}_{n}(\mathbb{F})$. Then

$$
C_{\sigma}(\mathbb{F})=\left[c_{n}\right]_{I_{\sigma}}^{c_{n}}(\mathbb{F}),
$$

where the principal order ideal $I_{\sigma}$ of $\left(c_{n}\right)^{c_{n}}$ is defined by

$$
I_{\sigma}:=\left(\{\sigma(1)\}_{<},\{\sigma(1), \sigma(2)\}_{<}, \ldots,\{\sigma(1), \sigma(2), \ldots, \sigma(n)\}_{<}\right)^{\downarrow}
$$

and $\left\{x_{1}, \ldots, x_{h}\right\}_{<} \in[n]_{<}^{h}$ is the tuple obtained ordering $x_{1}, \ldots, x_{h}$. 
Proof. A Schubert cell $C_{\sigma}(\mathbb{F})$ in $\phi_{c_{n}}\left(\mathrm{Fl}_{n}(\mathbb{F})\right)$ is an orbit of the flag $\phi_{c_{n}}\left(F_{\sigma}\right)$ under the action of $\pi_{Q}\left(I^{*}\left(c_{n} ; \mathbb{F}\right)\right)$. By Theorem [5.2, the group $\pi_{Q}\left(I^{*}\left(c_{n} ; \mathbb{F}\right)\right)$ is a subgroup of $I^{*}\left(\left(c_{n}\right)^{c_{n}} ; \mathbb{F}\right)$ and we conclude as in the proof of Proposition 4.16 .

We define the set of $Q$-Schubert cells of $\mathrm{Fl}_{\mathrm{P}}(\mathbb{F})$ as

$$
Q_{B}^{P}(\mathbb{F}):=\left\{I \in \mathcal{J}\left(Q^{P}\right):[Q]_{I}^{P}(\mathbb{F}) \neq \varnothing\right\} .
$$

Definition 5.6. Let $P, Q \in \operatorname{POS}(n)$. We call $\left(Q_{B}^{P}(\mathbb{F}), \subseteq\right)$ the $Q$-Bruhat poset of $\mathrm{Fl}_{\mathrm{P}}(\mathbb{F})$.

By Propositions 5.4 and 5.5 , the $c_{n}$-Bruhat poset of $\mathrm{Fl}_{n}(\mathbb{F})$ is isomorphic to $S_{n}$ with the Bruhat order.

In order to characterize the $Q$-Schubert cells of $\mathrm{Fl}_{\mathrm{P}}(\mathbb{F})$ we need to introduce a Gale order on the underlying set of $Q^{P}$.

\subsection{P-flags and the Maximality Property}

Let $P \in \operatorname{POS}(n)$ and define the set

$$
[n]^{P}:=\prod_{i=1}^{n}[n]_{<}^{\left|i^{\downarrow}\right|} .
$$

For example, we have that $[n]^{t_{n}} \simeq[n]^{n}$. The symmetric group $S_{n}$ acts on $[n]^{P}$ by setting

$$
\begin{gathered}
\sigma\left(\left(i_{1,1}\right),\left(i_{2,1}, \ldots, i_{2, \mid 2 \downarrow}\right), \ldots,\left(i_{n, 1}, \ldots, i_{n, \mid n \downarrow}\right)\right):= \\
\left(\left\{\sigma\left(i_{1,1}\right)\right\}_{<},\left\{\sigma\left(i_{2,1}\right), \ldots, \sigma\left(i_{2, \mid 2 \downarrow}\right)\right\}_{<}, \ldots,\left\{\sigma\left(i_{n, 1}\right), \ldots, \sigma\left(i_{n, \mid n \downarrow}\right)\right\}_{<}\right),
\end{gathered}
$$

for all $\sigma \in S_{n}$.

We introduce the following useful order on $[n]^{P}$.

Definition 5.7. The Gale ordering $\leqslant_{P}^{\sigma}$ on $[n]^{P}$ is defined by letting

$$
a \leqslant_{P}^{\sigma} b \Longleftrightarrow \sigma a \leqslant_{\left(c_{n}\right)^{P}} \sigma b,
$$

for all $a, b \in[n]^{P}$.

In particular, $\left([n]^{P}, \leqslant_{P}^{e}\right)=\left(c_{n}\right)^{P}$ and the Gale ordering $\leqslant_{t_{n}}^{e}$ on $[n]^{t_{n}}$ is $[n]^{n}$ ordered componentwise.

Remark 5.8. As in the proof of Proposition 4.10, we have that $Q^{P} \hookrightarrow\left(c_{n}\right)^{P}$, for all $P \in \operatorname{POS}(n)$. In particular, $Q^{P} \hookrightarrow\left([n]^{P}, \leqslant_{P}^{e}\right)$.

The following definitions are crucial for the study of incidence stratifications of a $P$-flag space, see Section 5.2 .

Definition 5.9. A subset $\mathcal{F} \subseteq[n]^{P}$ has the Maximality Property if the poset $\left(\mathcal{F}, \leqslant_{P}^{\sigma}\right)$ has maximum for all $\sigma \in S_{n}$.

Remark 5.10. By Remark [2.12, if $\mathcal{F} \subseteq[n]^{P}$ has the Maximality Property, then the poset $\left(\mathcal{F}, \leqslant_{P}^{\sigma}\right)$ has minimum for all $\sigma \in S_{n}$. 
Remark 5.11. It should be clear that, by definition, given $\mathcal{F} \subseteq[n]^{P}$ with the Maximality Property, the set $M_{i}:=\left\{F_{i}: F \in \mathcal{F}\right\}$ is a matroid of rank $\left|i^{\downarrow}\right|$, for all $i \in[n]$.

Recall from Section 2.3 that $M(W)$ stands for the matroid represented by the vector space $W$.

Definition 5.12. We say that $\mathcal{G} \subseteq[n]^{P}$ is represented by a $P$-flag $F \in \operatorname{Fl}_{\mathrm{P}}(\mathbb{F})$ if $\mathcal{G}=M\left(F_{1}\right) \times \ldots \times M\left(F_{n}\right)$.

As usual we identify $\biguplus_{k=0}^{n}[n]_{<}^{k}$ with the power set $\mathcal{P}([n])$. We define the following subset of $[n]^{P}$ :

$$
[n]_{\subseteq}^{P}:=\left\{a \in[n]^{P}: i<_{P} j \Rightarrow a_{i} \subsetneq a_{j}, \forall i, j \in[n]\right\} .
$$

Theorem 5.13. Let $\mathcal{G} \subseteq[n]^{P}$ be represented by a $P$-flag $F \in \mathrm{Fl}_{\mathrm{P}}(\mathbb{F})$. Then $\mathcal{G}$ has the Maximality Property and its $\leqslant_{P}^{\sigma}$-maximum lies in $[n]_{\subseteq}^{P}$, for all $\sigma \in S_{n}$.

Proof. By Definition [5.12, $\mathcal{G}_{h}=M\left(F_{h}\right)$ is a matroid, for every $h \in[n]$. Let $\sigma \in S_{n}$ and $m_{h}^{\sigma} \in \mathcal{G}_{h}$ be the maximum of the poset $\left(\mathcal{G}_{h}, \leqslant^{\sigma}\right)$, for all $h \in[n]$. It is clear that $\left(m_{1}^{\sigma}, \ldots, m_{n}^{\sigma}\right)$ is the maximum of $\left(\mathcal{G}, \leqslant_{P}^{\sigma}\right)$.

Let $i<_{P} j$. Then $\left|i^{\downarrow}\right|<\left|j^{\downarrow}\right|$ and $\left(F_{i}, F_{j}\right)$ is a partial flag; by [4, Theorem 1.7.3], the matroids $M\left(F_{i}\right)$ and $M\left(F_{j}\right)$ are concordant (see [4, Section 1.7.3]). By [4, Corollary 1.7.2], the pair $\left(m_{i}^{\sigma}, m_{j}^{\sigma}\right)$ satisfies $m_{i}^{\sigma} \subsetneq m_{j}^{\sigma}$, for all $\sigma \in S_{n}$.

The next result provides a fundamental tool to describe the $t_{n}$-stratification of a $P$-flag space, see Corollary 5.23 .

Proposition 5.14. Let $\mathcal{F}, \mathcal{G} \subseteq[n]^{P}$ represented by $P$-flags. Then

$$
\mathcal{F} \cap[n]_{\subseteq}^{P}=\mathcal{G} \cap[n]_{\subseteq}^{P} \Longrightarrow \mathcal{F}=\mathcal{G} .
$$

Proof. By contradiction, assume $\mathcal{F} \neq \mathcal{G}$ and let $m \in \mathcal{F}_{i} \backslash \mathcal{G}_{i}$, for some $i \in[n]$. Then there exists $\sigma \in S_{n}$ such that $m=\max \left(\mathcal{F}_{i}, \leqslant^{\sigma}\right)$. By Theorem 5.13 there exists $a:=\max \left(\mathcal{F}, \leqslant_{P}^{\sigma}\right) \in[n]_{\subseteq}^{P}$ and, by hypothesis, $a \in \mathcal{G}$. Hence $m=a_{i} \in \mathcal{G}_{i}$, a contradiction.

\section{$5.2 \quad Q$-stratification of $P$-flag spaces}

Now we are able to provide a characterization of $Q$-Schubert cells in the space $\mathrm{Fl}_{\mathrm{P}}(\mathbb{F})$.

Theorem 5.15. Let $P, Q \in \operatorname{POS}(n)$ and $I \in \mathcal{J}\left(Q^{P}\right)$. Then $[Q]_{I}^{P}(\mathbb{F}) \neq \varnothing$ if and only if there exists $I^{\prime} \subseteq I$ such that $\max (I) \cup I^{\prime}$ is represented by some $F \in \mathrm{Fl}_{\mathrm{P}}(\mathbb{F})$.

Proof. Let $I^{\prime} \subseteq I$ be any subset. The result follows by observing that $\max (I) \cup$ $I^{\prime}=M\left(F_{1}\right) \times \ldots \times M\left(F_{n}\right)$ for some $F \in \mathrm{Fl}_{\mathrm{P}}(\mathbb{F})$ if and only if there exists $A \in I^{*}\left(Q^{P} ; \mathbb{F}\right)$ such that

$$
A\left(\sum_{i \in \max (I)} e_{i_{1}} \otimes \ldots \otimes e_{i_{n}}\right)=\sum_{i \in \max (I) \cup I^{\prime}} a_{i} e_{i_{1}} \otimes \ldots \otimes e_{i_{n}}=\phi_{P}(F)
$$


where $a_{i} \in \mathbb{F} \backslash\{0\}$ for all $i \in \max (I)$ and we have defined

$$
e_{x}:=e_{x_{1}} \wedge \ldots \wedge e_{x_{k}}
$$

for all $x \in[n]_{<}^{k}$.

As a consequence of Theorem 5.15 we recover the Bruhat order of $S_{n}$.

Proposition 5.16. We have that $\left(S_{n}, \leqslant\right) \simeq\left([n]_{\subseteq}^{c_{n}}, \leqslant\left(c_{n}\right)^{c_{n}}\right) \simeq\left(\left(c_{n}\right)_{B}^{c_{n}}(\mathbb{F}), \subseteq\right)$.

Proof. The first poset isomorphism is clear by definition. Let $I_{1}, I_{2} \in \mathcal{J}\left(\left(c_{n}\right)^{c_{n}}\right)$ such that $I_{1} \subseteq I_{2}$ and $\max \left(I_{1}\right) \cup I_{1}^{\prime}, \max \left(I_{2}\right) \cup I_{2}^{\prime}$ are represented by flags, for some subsets $I_{1}^{\prime} \subseteq I_{1}, I_{2}^{\prime} \subseteq I_{2}$. Since the $c_{n}$-Gale order $\leqslant_{c_{n}}^{e}$ on $[n]^{c_{n}}$ is $\left(c_{n}\right)^{c_{n}}$, by the Maximality Property, $\left|\max \left[\max \left(I_{1}\right) \cup I_{1}^{\prime}\right]\right|=\left|\max \left(I_{1}\right)\right|=1$ and $\mid \max \left[\max \left(I_{2}\right) \cup\right.$ $\left.I_{2}^{\prime}\right]|=| \max \left(I_{2}\right) \mid=1$. By Theorem 5.13 we have that $\max \left(I_{1}\right), \max \left(I_{2}\right) \in[n]_{\subset}^{c_{n}}$. Moreover, if $\max (I) \in[n]_{\subset}^{c_{n}}$ then $\max (I)$ is clearly represented by a flag. Hence, by Theorem 5.15, $I \in\left(c_{n}\right)_{B}^{c_{n}}(\mathbb{F})$.

The following example shows the stratification of $\mathrm{Fl}_{3}(\mathbb{F})$ induced by the action on the projective space $\mathbb{P}[V \otimes(V \wedge V)]$ of the group $I^{*}\left(Q \times Q_{<}^{2} ; \mathbb{F}\right)$, where $Q \in \operatorname{POS}(3)$ is one of the posets of Example 3.14. In this case the factor $V \wedge V \wedge V$ is redundant.

Example 5.17. Let $P=c_{3}$ and $Q \in \operatorname{POS}(3)$ be the poset whose cover relations are $1 \triangleleft_{Q} 3$ and $2 \triangleleft_{Q} 3$. Then the poset $Q \times Q_{<}^{2}$ has the following Hasse diagran 5 :

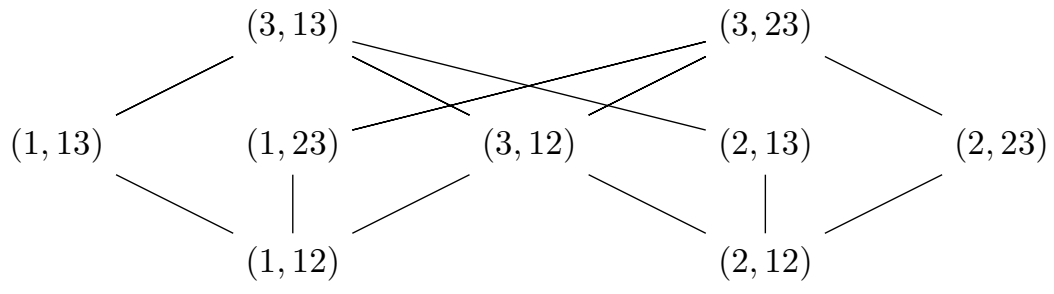

By Remark 5.8 and Theorem 5.13, the principal order ideals of $Q \times Q_{<}^{2}$ which satisfy the condition of Theorem 5.15 are the ones with maximum in the set

$$
\{(1,12),(2,12),(1,13),(2,23),(3,13),(3,23)\},
$$

which corresponds to the symmetric group $S_{3}$. We consider $S_{3}$ with its standard Coxeter presentation with generators $\{s, t\}$. Then $s=213=(2,12), t=132=$ $(1,13), s t=231=(2,23), t s=312=(3,13)$ and $s t s=321=(3,23)$.

Using Theorem 5.13, the non-principal order ideals to be considered are $\{(1,12),(2,12)\}$, which is represented by the flag

$$
\left(\operatorname{span}_{\mathbb{F}}\left\{e_{1}+e_{2}\right\}, \operatorname{span}_{\mathbb{F}}\left\{e_{1} \wedge e_{2}\right\}\right),
$$

and the order ideal $I=\{(1,13),(1,23),(2,13),(2,23)\}^{\downarrow}$. We have that $\max (I)$ is represented by the flag

$$
\left(\operatorname{span}_{\mathbb{F}}\left\{e_{1}+e_{2}\right\}, \operatorname{span}_{\mathbb{F}}\left\{\left(e_{1}+e_{2}\right) \wedge e_{3}\right\}\right) .
$$

Therefore the poset $\left(Q_{B}^{c_{n}}, \subseteq\right)$ has the following Hasse diagram:

\footnotetext{
${ }^{5}$ We omit parentheses when writing the elements of $[n]_{<}$and $[n]_{<}^{2}$.
} 


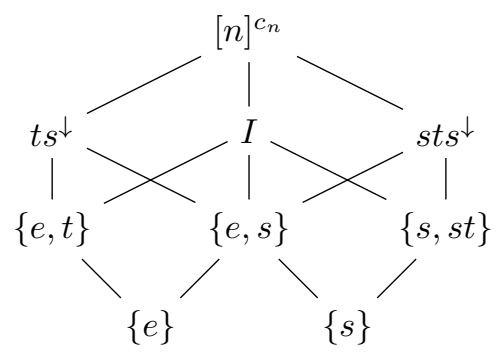

Notice that the action of $S_{n}$ on $[n]^{P}$ restricts to an action on $[n]_{\subseteq}^{P}$. We are ready to introduce the notion of $P$-flag matroid which extends the one of flag matroid, see Definition 2.14.

Definition 5.18. A subset $\mathcal{F} \subseteq[n]_{\subseteq}^{P}$ is a $P$-flag matroid if it has the Maximality Property.

The set $[n]_{\subseteq}^{P}$ is a $P$-flag matroid, which we call uniform $P$-flag matroid. Notice that $c_{n}$-flag matroids coincide with flag matroids in $S_{n}$.

Example 5.19. The uniform $t_{2}$-matroid is $[2]^{2}=\{(1,1),(1,2),(2,1),(2,2)\}$. We list all the $t_{2}$-matroids $\mathcal{F} \subsetneq[2]^{2}$ :

1. $|\mathcal{F}|=1:\{(1,1)\},\{(1,2)\},\{(2,1)\},\{(2,2)\}$.

2. $|\mathcal{F}|=2$ : $\{(1,1),(1,2)\},\{(1,1),(2,1)\},\{(1,1),(2,2)\},\{(1,2),(2,2)\}$, $\{(2,1),(2,2)\}$.

3. $|\mathcal{F}|=3:\{(1,1),(1,2),(2,2)\},\{(1,1),(2,1),(2,2)\}$.

For instance, the set $\{(1,2),(2,1)\}$ is not a $t_{2}$-matroid.

Definition 5.20. A P-flag matroid $\mathcal{F}$ is called representable over $\mathbb{F}$, if there exists $\mathcal{G} \subseteq[n]^{P}$ represented by $F \in \mathrm{Fl}_{\mathrm{P}}(\mathbb{F})$ such that $\mathcal{F}=\mathcal{G} \cap[n]_{\subseteq}^{P}$.

Example 5.21. Since $[n]_{\subset}^{t_{n}}=[n]^{t_{n}}$, then $\left(\left(t_{n}\right)_{B}^{t_{n}}(\mathbb{F}), \subseteq\right)$ is the poset of $\mathbb{F}$ representable $t_{n}$-flag matroi $\bar{d} s$. The $\mathbb{F}$-representable $t_{2}$-flag matroids are $\{(1,2)\}$, $\{(2,1)\},\{(1,1),(1,2)\},\{(1,1),(2,1)\},\{(1,2),(2,2)\},\{(2,1),(2,2)\}$ and the uniform one. The Hasse diagram of $\left(t_{2}\right)_{B}^{t_{2}}$ is

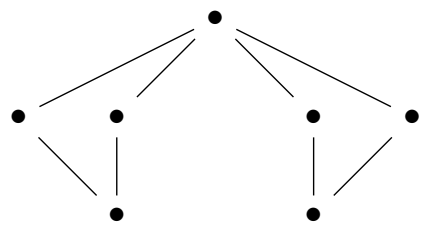

The following results extend the flag matroid stratification of a flag variety.

Proposition 5.22. Let $P \in \operatorname{POS}(n)$ and $I, J \in \mathcal{J}\left(\left(t_{n}\right)^{P}\right)$ such that $\left[t_{n}\right]_{I}^{P}(\mathbb{F}) \neq$ $\varnothing$ and $\left[t_{n}\right]_{J}^{P}(\mathbb{F}) \neq \varnothing$. Then

$$
I \cap[n]_{\subseteq}^{P}=J \cap[n]_{\subseteq}^{P} \Longrightarrow\left[t_{n}\right]_{I}^{P}(\mathbb{F})=\left[t_{n}\right]_{J}^{P}(\mathbb{F}) .
$$


Proof. By Theorem [5.15], we have that $I=\max (I)$ and $J=\max (J)$ are represented by some $P$-flags $F$ and $G$, respectively. The result follows by Proposition 5.14

Corollary 5.23. The set $\left(t_{n}\right)_{B}^{P}(\mathbb{F})$ is in bijection with the set of $\mathbb{F}$-representable $P$-flag matroids.

Proof. Let $I \in \mathcal{J}\left(\left(t_{n}\right)^{P}\right)$. By Theorem [5.15, $I \in\left(t_{n}\right)_{B}^{P}(\mathbb{F})$ if and only if it is represented by a $P$-flag. By Theorem 5.13, $I$ has the Maximality Property. Then $I \cap[n]_{\subset}^{P}$ is a $P$-flag matroid and, by Definition [5.20, it is representable over $\mathbb{F}$. Hence the result follows by Proposition 5.22 .

We conclude with the following conjecture.

Conjecture 5.24. Let $n>0$ and $Q, P \in \operatorname{POS}(n)$. Then the poset $\left(Q_{B}^{P}(\mathbb{C}), \subseteq\right)$ is graded.

By Proposition 5.16, when $Q=P=c_{n}$ Conjecture 5.24 holds, since the Bruhat order on $S_{n}$ is graded. Also for $P=t_{n}$ and $Q=c_{n}$ the poset is graded, see Corollary 5.31

\subsection{The $t_{n}$-flag space and its parking function stratifica- tion}

In this section we provide an incidence stratification of a $t_{n}$-flag space by parking functions. We refer to [28, Exercise 5.49], 30] and [33] for further details and references on parking functions.

Definition 5.25. A parking function over $n$ is an element $a \in[n]^{n}$ such that $\left(a_{1}, \ldots, a_{n}\right) \leqslant_{c_{n}^{n}}(\sigma(1), \ldots, \sigma(n))$, for some permutation $\sigma \in S_{n}$.

For example $(4,1,1,1,2,6,4)$ is a parking function over 7 whereas the element $(6,6,6,1,2,3,4)$ is not a parking function.

Definition 5.26. Let $a \in[n]^{n}$. If $a \geqslant_{c_{n}^{n}}(\sigma(1), \ldots, \sigma(n))$ for some $\sigma \in S_{n}$, we say that $a$ is a dual parking function over $n$.

For example $(6,3,5,1,2,7,7)$ is a dual parking function over 7 whereas the element $(1,2,2,2,2,4,3)$ is not a dual parking function. Notice that the self-dual parking functions are the permutations.

In the following theorem we describe the $c_{n}$-stratification of the space $\mathrm{Fl}_{t_{n}}(\mathbb{F})$.

Theorem 5.27. Let $I$ be an order ideal of $\left(c_{n}\right)^{t_{n}}$. Then $\left[c_{n}\right]_{I}^{t_{n}}(\mathbb{F}) \neq \varnothing$ if and only if $|\max (I)|=1$ and $\max (I)$ is a dual parking function.

Proof. Notice that subsets of $[n]^{t_{n}}$ represented by $t_{n}$-flags coincide with representable $t_{n}$-flag matroids. By Remark [5.8, only principal order ideals have to be considered in Theorem 5.15, for the other ones have more than one maximal element.

A representable $t_{n}$-flag matroid over $\mathbb{F}$ is represented by an element $v:=v_{1} \otimes$ $\ldots \otimes v_{n} \in \bigotimes_{i=1}^{n} \mathbb{F}^{n}$, such that $v_{1}, \ldots, v_{n}$ are linearly independent or, equivalently, by a matrix $M(v) \in \mathrm{GL}(n, \mathbb{F})$ with columns $v_{1}, \ldots, v_{n}$. Therefore

$$
v=\sum_{i \in[n]^{n}}\left(\left(v_{1}\right)_{i_{1}} \cdots\left(v_{n}\right)_{i_{n}}\right) e_{i_{1}} \otimes \ldots \otimes e_{i_{n}},
$$


where $\left(v_{i}\right)_{j} \in \mathbb{F}$ is the $j$-th component of the vector $v_{i}$, for all $i, j \in[n]$. It is clear that, since $M(v) \in \mathrm{GL}(n, \mathbb{F})$, there exists $\sigma \in S_{n}$ such that $\left(v_{1}\right)_{\sigma(1)} \cdots\left(v_{n}\right)_{\sigma(n)} \neq$ 0 .

If $\left[c_{n}\right]_{I}^{t_{n}}(\mathbb{F}) \neq \varnothing$ then, by our previous considerations and Theorem $[5.15$, there exists $\sigma \in S_{n}$ such that $(\sigma(1), \ldots, \sigma(n)) \in I$. This implies $\max (I) \geqslant_{c_{n}^{n}}$ $(\sigma(1), \ldots, \sigma(n))$; so $\max (I)$ is a dual parking function.

On the other hand, if $\max (I)=a$ is a dual parking function then $a \geqslant_{c_{n}^{n}}$ $(\sigma(1), \ldots, \sigma(n))$ for some $\sigma \in S_{n}$ and the vector

$$
\begin{aligned}
v & :=\sum_{(\sigma(1), \ldots, \sigma(n)) \leqslant c_{n}^{n} b \leqslant c_{n}^{n} a} e_{b_{1}} \otimes \ldots \otimes e_{b_{n}} \\
& =\left(\sum_{\sigma(1) \leqslant i_{1} \leqslant a_{1}} e_{i_{1}}\right) \otimes \ldots \otimes\left(\sum_{\sigma(n) \leqslant i_{n} \leqslant a_{n}} e_{i_{n}}\right)
\end{aligned}
$$

represents over $\mathbb{F}$ a $t_{n}$-flag matroid, since the matrix $M(v)$ is equivalent to an invertible upper triangular matrix. Then the condition of Theorem 5.15 is satisfied.

Remark 5.28. The $t_{n}$-flag space has been stratified by permutations in [22] by gluing the orbits of the left action of the group of lower triangular matrices (more in general the varieties $X_{n, k}$ studied there have been stratified by Fubini words, which reduce to permutations when $k=n$ ).

Remark 5.29. An incidence stratification of $\mathrm{Fl}_{t_{n}}(\mathbb{F})$ made of parking function can be obtained by considering the action of the group of lower triangular matrices.

Remark 5.30. By Theorem 5.27, an analog of Corollary 4.22 for Q-Schubert cells of $\mathrm{Fl}_{\mathrm{P}}(\mathbb{F})$ does not hold.

Corollary 5.31. The poset $\left(\left(c_{n}\right)_{B}^{t_{n}}(\mathbb{F}), \subseteq\right)$ has cardinality $(n+1)^{n-1}$ and it is graded, with rank function $\rho(a):=\sum_{i=1}^{n}\left(a_{i}-i\right)$, for any dual parking function $a \in[n]^{n}$.

\section{Acknowledgements}

The first author was partially supported by Swiss National Science Foundation Professorship grant PP00P2_179110/1 of Prof. Emanuele Delucchi.

He is grateful to the town of Zagarolo, where this paper started and finished, for the hospitality received there.

\section{References}

[1] A. Björner and F. Brenti, Combinatorics of Coxeter Groups, Graduate Texts in Mathematics, 231, Springer-Verlag, New York, 2005.

[2] A. Björner et al., Oriented matroids, Vol. 46. Cambridge University Press, 1999. 
[3] J. Bonin, A. de Mier, and M. Noy, Lattice path matroids: enumerative aspects and Tutte polynomials, J. Comb. Theory Series A 104.1, 63-94 (2003).

[4] A. Borovik, I. M. Gelfand and N. White, Coxeter matroids, Birkhäuser, Progress in Mathematics, 216, 2003.

[5] M. Bousquet-Mélou and S. Butler, Forest-like permutations, Ann. Comb. 11, 335-354 (2007).

[6] M. Brandt and A. Wiebe, The slack realization space of a matroid, Algebraic Comb., 2.4, 663-681 (2019).

[7] M. Brion, Lectures on the geometry of flag varieties, Topics in cohomological studies of algebraic varieties. Birkhäuser Basel, 33-85, 2005.

[8] F. Caselli, M. D'Adderio and M. Marietti, Weak generalized lifting property, Bruhat intervals and Coxeter matroids, Int. Math. Res. Not. 2021.3, 16781698 (2021).

[9] G. Chatel, V. Pilaud and V. Pons, The weak order on integer posets, Algebraic Comb., 2.1, 1-48 (2019).

[10] W. E. Duckworth, Infiniteness of double coset collections in algebraic groups, J. of Algebra 273.2, 718-733 (2004).

[11] H. Flaschka and L. Haine, Torus orbits in G/P, Pacific J. Math. 149.2, 251-292 (1991).

[12] N. Ford, The expected codimension of a matroid variety, J. Algebr. Comb. 41.1, 29-47 (2015).

[13] J. Gandini and G. Pezzini, Orbits of strongly solvable spherical subgroups on the flag variety, J. Algebr. Comb. 47.3, 357-401 (2018).

[14] I. M. Gelfand, R. M. Goresky, R. D. MacPherson and V. V. Serganova, Combinatorial geometries, convex polyhedra, and Schubert cells, Advances in Math. 63.3, 301-316 (1987).

[15] J. Hilgert and K-H. Neeb, Structure and geometry of Lie groups, Springer Science \& Business Media, 2011.

[16] J. E. Humphreys, Reflection Groups and Coxeter Groups, Cambridge Studies in Advanced Mathematics, 1990.

[17] V. Lakshmibai and J. Brown, The Grassmannian variety, 42, Springer, New York, 2015.

[18] V. Lakshmibai and J. Brown, Flag varieties: an interplay of geometry, combinatorics, and representation theory, 53, Springer, 2018.

[19] S. Oh, Positroids and Schubert matroids, J. of Comb. Theory Series A 118.8, 2426-2435 (2011).

[20] S. Oh, A. Postnikov, and Hwanchul Yoo, Bruhat order, smooth Schubert varieties, and hyperplane arrangements, J. of Comb. Theory Series A 115.7, 1156-1166 (2008). 
[21] J. G. Oxley, Matroid theory, Vol. 3. Oxford University Press, USA, 2006.

[22] B. Pawlowski and B. Rhoades, A flag variety for the Delta Conjecture, Trans. of the Am. Math. Soc. 372.11, 8195-8248 (2019).

[23] B. Rhoades, Spanning subspace configurations, Sel. Math. New Ser. 27, 8 (2021).

[24] D. B. Rush and X. Shi, On orbits of order ideals of minuscule posets, J. Algebr. Comb. 37.3, 545-569 (2013).

[25] C.S. Seshadri, Introduction to the theory of standard monomial theory, Texts and Readings in Mathematics 46, Springer.

[26] E. Spiegel and Ch. O'Donnell, Incidence algebras, Vol. 206, CRC Press, 1997.

[27] R. P. Stanley, Enumerative Combinatorics, Vol. 1, Wadsworth and Brooks/Cole, Monterey, CA, 1986.

[28] R. P. Stanley, Enumerative Combinatorics, Vol. 2, Cambridge Studies in Advanced Mathematics, no. 62, Cambridge Univ. Press, 1999.

[29] B. Sturmfels, On the matroid stratification of Grassmann varieties, specialization of coordinates, and a problem of $N$. White, Advances in Math. 75.2, 202-211 (1989).

[30] S. Van Willigenburg, The shuffle conjecture, Bull. Amer. Math. Soc. 57.1, 77-89 (2020).

[31] N. L. White, The transcendence degree of a coordinatization of a combinatorial geometry, J. Comb. Theory Series B 29.2, 168-175 (1980).

[32] N. L. White eds, Theory of matroids, Cambridge University Press, No. 26, 1986.

[33] C. H. Yan, Parking functions, Handbook of Enumerative Combinatorics. Chapman and Hall/CRC, 859-918, 2015. 